\title{
Computations of the first eigenpairs for the Schrödinger operator with magnetic field
}

\author{
V. Bonnaillie-Noël, M. Dauge, D. Martin and G. Vial
}

\begin{abstract}
This paper is devoted to computations of eigenvalues and eigenvectors for the Schrödinger operator with constant magnetic field in a domain with corners, as the semi-classical parameter $h$ tends to 0 . The eigenvectors corresponding to the smallest eigenvalues concentrate in the corners: They have a two-scale structure, consisting of a corner layer at scale $\sqrt{h}$ and an oscillatory term at scale $h$. The high frequency oscillations make the numerical computations particularly delicate. We propose a high order finite element method to overcome this difficulty. Relying on such a discretization, we illustrate theoretical results on plane sectors, squares, and other straight or curved polygons. We conclude by discussing convergence issues.
\end{abstract}

\section{Introduction}

Superconductivity theory, modeled by Ginzburg and Landau, motivates investigations of the Schrödinger operator with magnetic field and Neumann boundary conditions in twodimensional domains. The Schrödinger operator $-(h \nabla-i \mathcal{A})^{2}$ derives from a linearization of the Ginzburg-Landau functional and the behavior of its eigenvalues and eigenvectors as $h \rightarrow 0$ gives information about the onset of superconductivity in the material, see [6, 7] 13, $14,20,29]$ for the general framework and [2, $15,16,17,[18,19,24,26,28]$ for more closely related questions concerning the Schrödinger operator.

We give the mathematical framework we will work within: let $\Omega$ denote a bounded polygonal domain in $\mathbb{R}^{2}$ and $\mathcal{A}$ the magnetic potential $\frac{1}{2}\left(-x_{2}, x_{1}\right)$ defined on $\mathbb{R}^{2}$. We investigate the behavior of the eigenpairs of the Neumann realization $P_{h}$ on $\Omega$ for the Schrödinger operator $-(h \nabla-i \mathcal{A})^{2}$ as $h \rightarrow 0$. The variational space associated with $P_{h}$ is $H^{1}(\Omega)$ and its domain is the subspace of functions $u$ such that $P_{h} u \in L^{2}(\Omega)$ and $\nu \cdot(h \nabla-i \mathcal{A}) u=0$ on $\partial \Omega$, with $\nu$ denoting the unit normal to $\partial \Omega$.

Let us first mention that the Schrödinger operator $P_{h}$ is gauge invariant in the sense of the following proposition:

Proposition 1.1. Let $\phi \in H^{2}(\Omega)$, then $u$ is an eigenvector associated with the eigenvalue $\mu$ for the operator $-(h \nabla-i \mathcal{A})^{2}$ if and only if $u_{\phi}:=\mathrm{e}^{i \phi / h} u$ is an eigenvector associated with the eigenvalue $\mu$ for the operator $-(\nabla-i(\mathcal{A}+\nabla \phi))^{2}$. 
In particular, the eigenvalues of the Schrödinger operator are the same for any potential $\tilde{\mathcal{A}}$ such that $\operatorname{curl} \tilde{\mathcal{A}}=\operatorname{curl} \mathcal{A}$. This allows the use of adapted gauges according to the domain.

In [10], a complete asymptotic expansion of low-lying eigenstates is exhibited for curvilinear polygonal domains and refined results are proved in the case when the domain has straight sides and the magnetic field is constant. The eigenmodes have a two-scale structure, in the form of the product of a corner layer at scale $\sqrt{h}$ with an oscillatory term at scale $h$. The latter makes the numerical approximation delicate. A posteriori error estimates are used in [3 9] to determine localized mesh refinement in a low degree finite element method. We investigate here a finite element method using high degree polynomials, as described in Section 2,

It is proved in [10] that the study of the Schrödinger operator $P_{h}$ in a domain with corners of openings $\alpha_{1}, \ldots, \alpha_{J}$, relies on those of the Schrödinger operator $Q^{\alpha}:=-(\nabla-i \mathcal{A})^{2}$ on an infinite sector of opening $\alpha$, for $\alpha=\alpha_{1}, \ldots, \alpha_{J}$. Section 3 is devoted to this operator: We show computations which make theoretical results more complete.

The next sections deal with the asymptotic behavior of the eigenstates of $P_{h}$ as $h$ goes to 0 : We give numerical solutions which illustrate the clustering of eigenvalues, depending on the symmetries of the domain. Several particular polygonal domains are investigated, highlighting different points of the theory: Tunneling effect for the square, concentration in the lowest corners for the trapezoid, the rhombus or the L-shaped domain. We end with a curvilinear polygon for which the asymptotics is appreciably different.

We conclude the paper in Section 7 by numerical error curves for the specific case of a standard square of length 2 , and $h=0.02$. We compare the performances of "pextensions" (increasing the polynomial degree on a fixed mesh), and of "h-extensions" (refining the mesh with a fixed degree). According to the magnitude of $h$, a locking phenomenon is present, stronger and stronger as $h \rightarrow 0$. A disturbing feature of this locking is the preasymptotic convergence to interior modes, corresponding to the lowest Landau level, significantly larger than the correct eigenvalues. Our conclusion is the necessity for using "p-extensions" if we wish to capture fine effects like the tunneling effect in symmetric domains.

\section{General results on eigenvalue approximation}

In the sequel, we will show numerical results of spectral approximations for the Schrödinger operator in various domains. We wish first to recall some facts on the numerical computation of eigenvalues and eigenvectors by a finite element Galerkin method, which serve as a basis to justify the relevance of our results.

Let us fix some notation:

- $\mu_{h, n}$ is the $n$-th eigenvalue of the operator $P_{h}$,

- $u_{h, n}$ is a normalized associated eigenfunction in $\mathcal{V}=H^{1}(\Omega)$,

- $\left(\mathcal{T}^{\ell}\right)_{\ell>0}$ is a family of quadrilateral meshes, where $\ell$ is the maximum size of the elements (we changed the traditional $h$ into $\ell$ since the letter $h$ already stands for the 
small semi-classical parameter),

- $\mathbb{Q}_{p}$ is the standard space of polynomials of partial degree $p$ in the reference square element,

- $\mathcal{V}^{\ell, p}$ is the conforming discrete variational space associated with the $\mathbb{Q}_{p}$-reference square element on the mesh $\mathcal{T}_{\ell}$,

- $\left(\mu_{h, n}^{\ell, p}, u_{h, n}^{\ell, p}\right)$ is the $n$-th discrete eigenpair of $P_{h}$ in $\mathcal{V}^{\ell, p}$ :

$$
\int_{\Omega}(h \nabla-i \mathcal{A}) u_{h, n}^{\ell, p} \cdot \overline{(h \nabla-i \mathcal{A}) v} d x=\mu_{h, n}^{\ell, p} \int_{\Omega} u_{h, n}^{\ell, p} \bar{v} d x, \quad \forall v \in \mathcal{V}^{\ell, p} .
$$

For the first eigenpair ( $n=1$ ) or, more generally, if $\mu_{h, n} \neq \mu_{h, n-1}$, it is known from [4] 5 , 11] that the following Céa-like estimate holds

$$
\left|\mu_{h, n}-\mu_{h, n}^{\ell, p}\right| \leq L_{h, n}^{\ell, p} \sup _{u \in M_{h, n}} \inf _{\chi \in \mathcal{V}^{\ell, q}}\|u-\chi\|_{\mathcal{V}}^{2},
$$

where $M_{h, n}$ is the set of normalized eigenvectors 1 associated with $\mu_{h, n}$ and $L_{h, n}^{\ell, p}$ a positive constant which, for each fixed $h>0$ and $n \in \mathbb{N}$, is bounded as $\ell \rightarrow 0$ or $p \rightarrow \infty$. Moreover the corresponding estimate for eigenvectors reads: There exists an eigenvector $\tilde{u}_{h, n}$ associated with $\mu_{h, n}$ satisfying

$$
\left\|\tilde{u}_{h, n}-u_{h, n}^{\ell, p}\right\|_{\mathcal{V}} \leq L_{h, n}^{\ell, p} \sup _{u \in M_{h, n}} \inf _{\chi \in \mathcal{V}^{\ell, q}}\|u-\chi\|_{\mathcal{V}}
$$

Thus discretization errors on the eigenpairs are essentially bounded by the best approximation errors on the eigenvectors of $P_{h}$. We have to keep in mind that the latter closely depends on the semi-classical parameter $h$.

In the following, we will interpret the Galerkin approximations obtained for the eigenpairs, with respect to the asymptotic results of [10]. We emphasize the fact that, since by construction $\mathcal{V}^{\ell, p} \subset \mathcal{V}$, the computed eigenvalues will always be greater than the exact eigenvalue of same rank.

All the results displayed in this paper have been obtained with the Finite Elements Library Mélina, see [27]. Computations are mostly done with pretty coarse meshes (consisting of less than 100 quadrilaterals), but with high polynomial degree (10 in general, referred to as $\mathbb{Q}_{10}$-approximation). We justify our choice of a "p-extension" (where the degree $p$ of polynomials is increased), rather than a "h-extension" (where the size $\ell$ of the elements is decreased), by the fact that - for the same number of degrees of freedom - a p-extension captures oscillations more accurately than a h-extension, see [1, 22, 23] for related questions concerning the Helmholtz equation and dispersion relations at high wave number. This point is discussed in more detail in Section 7

\section{Model operators in infinite sectors}

This section is devoted to the study of the Schrödinger operator $-(\nabla-i \mathcal{A})^{2}$ in an infinite sector: The analysis of the operator $P_{h}$ in a bounded domain with corners relies on this

\footnotetext{
${ }^{1}$ If $\mu_{h, n}=\mu_{h, n-1}$, the set $M_{h, n}$ has to be modified accordingly.
} 
model situation. We first recall some theoretical results from [8] concerning the spectrum of the operator and, next, we show some numerical experiments which illustrate some of these results or give hints on how to extend them.

\subsection{Theoretical results on sectors}

We denote by $\mathrm{X}=\left(\mathrm{X}_{1}, \mathrm{X}_{2}\right)$ the Cartesian coordinates in $\mathbb{R}^{2}$, and by $R=|\mathrm{X}|$ and $\theta$ the polar coordinates. Let $G^{\alpha}$ be the sector in $\mathbb{R}^{2}$ with opening $\alpha$ :

$$
G^{\alpha}=\left\{\mathrm{X} \in \mathbb{R}^{2}, \quad \theta \in(0, \alpha)\right\},
$$

and $Q^{\alpha}$ be the Neumann realization of the Schrödinger operator $-(\nabla-i \mathcal{A})^{2}$ on the sector $G^{\alpha}$. With the potential $\mathcal{A}(\mathrm{X})=\frac{1}{2}\left(-\mathrm{X}_{2}, \mathrm{X}_{1}\right)$, the operator $Q^{\alpha}$ takes the form

$$
Q^{\alpha}=-\Delta+i\left(\mathrm{X}_{1} \partial \mathrm{X}_{2}-\mathrm{X}_{2} \partial \mathrm{X}_{1}\right)+\frac{1}{4}|\mathrm{X}|^{2}
$$

The operator $Q^{\alpha}$ is associated with the following variational space

$$
\mathcal{V}^{\alpha}=\left\{\Psi \in L^{2}\left(G^{\alpha}\right), \quad(\nabla-i \mathcal{A}) \Psi \in L^{2}\left(G^{\alpha}\right)\right\},\|\Psi\|_{\mathcal{V}^{\alpha}}^{2}=\|\Psi\|_{L^{2}}^{2}+\|(\nabla-i \mathcal{A}) \Psi\|_{L^{2}}^{2} .
$$

We denote by $\mu_{k}(\alpha)$ the $k$-th smallest element of the spectrum given by the max-min principle. We quote some results of [8] about the spectrum of $Q^{\alpha}$.

\section{Theorem 3.1.}

(i) The infimum of the essential spectrum of $Q^{\alpha}$ is equal to $\Theta_{0}:=\mu_{1}(\pi)$.

(ii) For all $\alpha \in(0, \pi / 2], \mu_{1}(\alpha)<\Theta_{0}$ and, therefore, $\mu_{1}(\alpha)$ is an eigenvalue.

(iii) Let $\alpha \in(0,2 \pi)$ and $k \geq 1$. Let $\Psi_{k}^{\alpha}$ be an eigenfunction associated with $\mu_{k}(\alpha)<\Theta_{0}$ for the operator $Q^{\alpha}$. Then $\Psi_{k}^{\alpha}$ satisfies the following exponential decay estimate:

$$
\forall \varepsilon>0, \exists C_{\varepsilon, \alpha}>0, \quad\left\|\mathrm{e}^{\left(\sqrt{\Theta_{0}-\mu_{k}(\alpha)}-\varepsilon\right)|\mathrm{X}|} \Psi_{k}^{\alpha}\right\|_{\mathcal{V}^{\alpha}} \leq C_{\varepsilon, \alpha}
$$

(iv) For all $\alpha \in(0, \pi]$,

$$
\frac{\Theta_{0}}{\pi} \leq \frac{\mu_{1}(\alpha)}{\alpha} \leq \frac{1}{\sqrt{3}}
$$

and there holds

$$
\frac{\mu_{1}(\alpha)}{\alpha} \rightarrow \frac{1}{\sqrt{3}} \quad \text { as } \quad \alpha \rightarrow 0 .
$$

Remark 3.2. Using the same technique as [8], one can establish asymptotics of the $k$-th eigenvalue as $\alpha \rightarrow 0$, similar to (5):

$$
\frac{\mu_{k}(\alpha)}{\alpha} \rightarrow \frac{2 k+1}{\sqrt{3}} \quad \text { as } \quad \alpha \rightarrow 0 .
$$




\subsection{Numerical experiments on sectors}

We present here some results of numerical computations of the low-lying eigenvalues, which illustrate the estimates (4), (5) and (6). Furthermore, it allows to investigate the monotonic behavior of $\mu_{k}(\alpha)$ with respect to the opening $\alpha$.

The method we have used to compute approximations of the eigenvalues consists in a high order finite element method, using quadrilateral elements and tensor-product polynomials of degree 10. Let us explain the way we deal with the unboundedness of the domain: For a given $\alpha$, we mesh a bounded cornered strip $\omega$ of opening $\alpha$, see Figure 1 and, for any $h>0$, we consider the scaled operator $Q_{h, \omega}^{\alpha}$ defined on $\omega$ as

$$
Q_{h, \omega}^{\alpha}=-(h \nabla-i \mathcal{A})^{2} .
$$
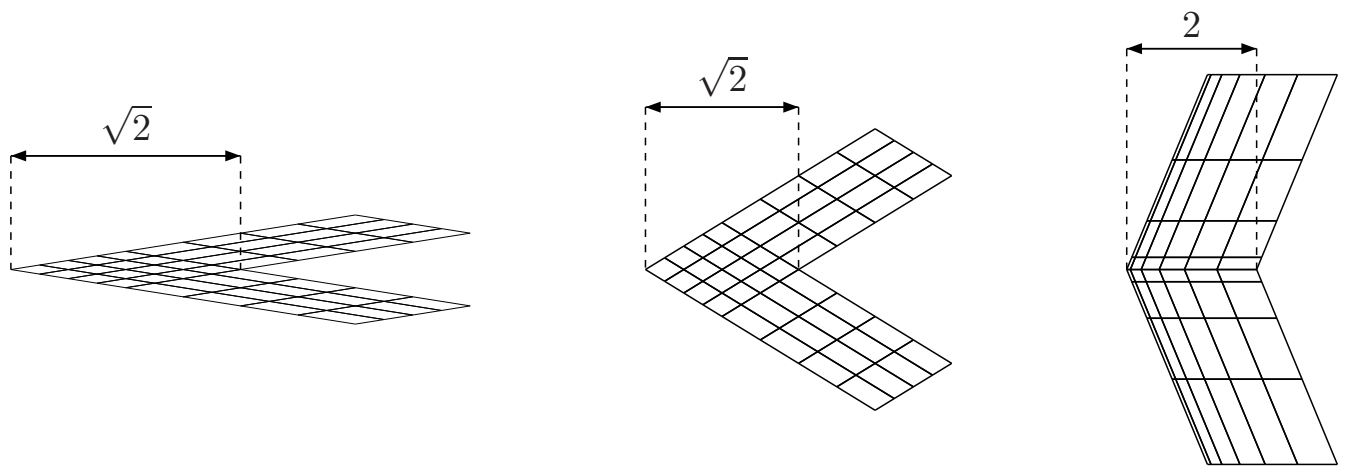

Figure 1: Meshes on cornered strips for $\alpha=0.1 \pi, 0.35 \pi, 0.75 \pi$.

By dilatation, the eigenvalues of the operator $Q_{1, h^{-1} \omega}^{\alpha}$ are the same as those of $Q_{h, \omega}^{\alpha}$ divided by $h$. Consequently, taking the decay of eigenvectors into account, we recover the eigenvalues of $Q^{\alpha}$ on the infinite sector $G^{\alpha}$ at the limit $h \rightarrow 0$. This formulation offers the advantage to be consistent with the analysis in the next sections for bounded domains.
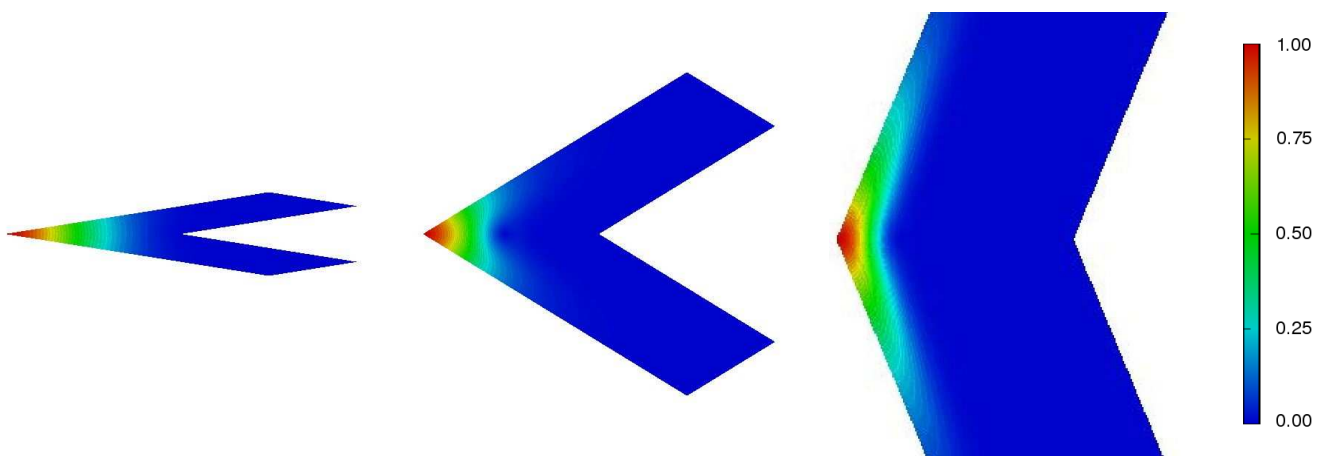

Figure 2: Moduli of the first eigenfunction for $\alpha=0.1 \pi, 0.35 \pi, 0.75 \pi$.

To ensure that the eigenvalues in the infinite sector are approximated from above, we impose Dirichlet boundary conditions on the edges of $\omega$ which differ from the boundary 
of $G^{\alpha}$ (keeping natural Neumann conditions elsewhere). The choice of meshes such as in Figure 1 is justified by the localization of the eigenvectors given in Theorem 3.1. This exponential concentration is illustrated in Figure 2. We note that the behavior of the first eigenvector changes when the opening increases. Indeed, when the opening is small (e.g., $\alpha \leq \pi / 10$ like in the left picture of Figure 2), the eigenvector appears to be essentially radial, in coherence with asymptotics as $\alpha \rightarrow 0$. When the opening increases, the modulus of the eigenvector spreads out along the boundary (see right picture of Figure 2). Consequently, we realize computations with two different meshes according to the opening (the mesh on the right of Figure 1 is refined near the edges where the eigenvector is expected to be mostly supported).

\subsubsection{Asymptotics of $\mu_{k}(\alpha)$ as $\alpha \rightarrow 0$}

In order to increase the accuracy of the approximation of the eigenvalues for small angles, we introduce a gauge transform which leads to the potential $\tilde{\mathcal{A}}(x)=\left(-x_{2}, 0\right)$. The resulting operator $\tilde{Q}^{\alpha}=-(\nabla-i \tilde{\mathcal{A}})^{2}$ has the same spectrum than $Q^{\alpha}$, as explained in Proposition 1.1. The relevance of such a transform is linked to the amplitude of the potential: for small openings $\alpha, \tilde{\mathcal{A}}$ is smaller than $\mathcal{A}$ in the considered domain. We expect a better approximation for $\tilde{Q}^{\alpha}$ than for $Q^{\alpha}$ since the associated eigenvectors are less oscillating.
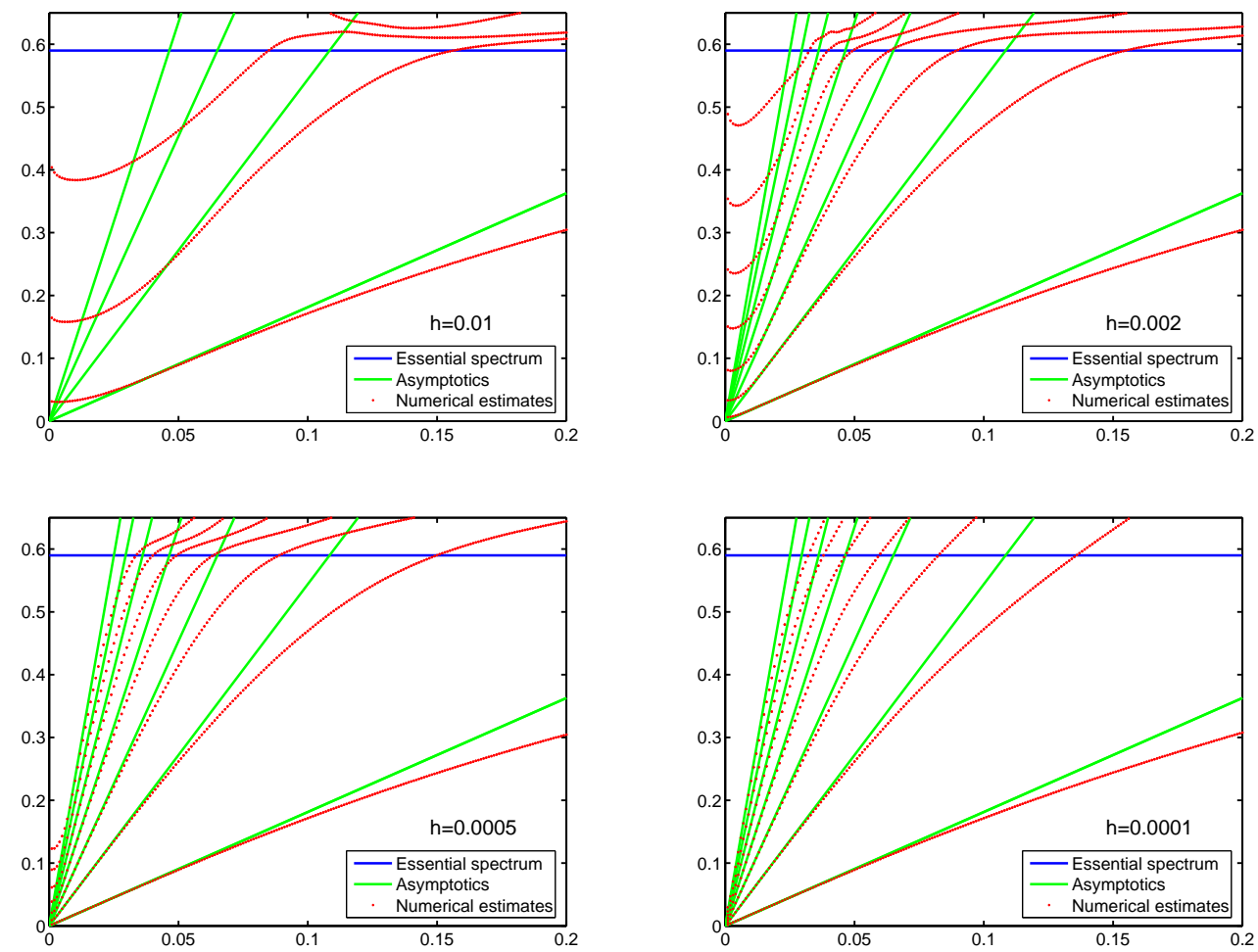

Figure 3: $\mu_{k}(\alpha)$ vs. $\frac{\alpha}{\pi}, k=1, \ldots, 7$.

In Figure 3 we present numerical computations of $\mu_{k}(\alpha)$ for $k=1, \cdots, 7$ and $\alpha \in$ 
$\{j \pi / 1000, j=1, \ldots, 200\}$ using $h=0.01,0.002,0.0005,0.0001$. We observe that we capture very precisely the asymptotics of $\mu_{k}(\alpha)$ given in (6) as soon as the parameter $h$ is small. This is a consequence of the behavior of the eigenvectors recalled in Theorem 3.1 the eigenfunctions are localized near the corner and are exponentially small far from the corner. Consequently, the less $h$, the less information we lose, and the better the approximation of the eigenpairs.

The improvement of the approximation for small angles is clear in Figures 3, whereas the situation seems to be the reverse for larger values of $\alpha$. Indeed, the eigenvalues being approximated from above, the results for $\alpha>\pi / 10$ are deteriorating for small $h$. This phenomenon can be explained by the fact that we keep the same number of elements to capture higher oscillations: the mesh is too coarse to approximate accurately the eigenfunctions.

\subsubsection{Monotonicity of $\alpha \mapsto \mu_{1}(\alpha)$}

Let us now focus on the first eigenvalue. We have observed the asymptotic behavior (4) as $\alpha \rightarrow 0$ in Figure 3 for $\alpha \in(0, \pi / 5)$. Figure 5 gives computations for $\alpha \in(0, \pi)$ with a discretization $\{j \pi / 200, j=1, \ldots, 200\}$. We have realized these computations with several values of $h$ between $10^{-4}$ and 0.5 and three magnetic potentials $\mathcal{A}(x)=\frac{1}{2}\left(-x_{2}, x_{1}\right)$ (symmetric gauge), $\widetilde{\mathcal{A}}(x)=\left(-x_{2}, 0\right)$ and $\widehat{\mathcal{A}}(x)=\left(0, x_{1}\right)$ (Landau gauges). According to Proposition 1.1 the Schrödinger operator associated with these three potentials have the same spectrum and the eigenvectors can be easily deduced one from the other. We show in Figure 4 the effect of the gauge on the phase of the first eigenvector. The potential $\widetilde{\mathcal{A}}$ is better adapted for small openings ( $\alpha<\pi / 10$ ), the potential $\widehat{\mathcal{A}}$ is more convenient for large openings $(\alpha>19 \pi / 20)$ since the eigenvector is localized in the corner and also along the Neumann boundary. For the other openings, the potential $\mathcal{A}$ gives better results.
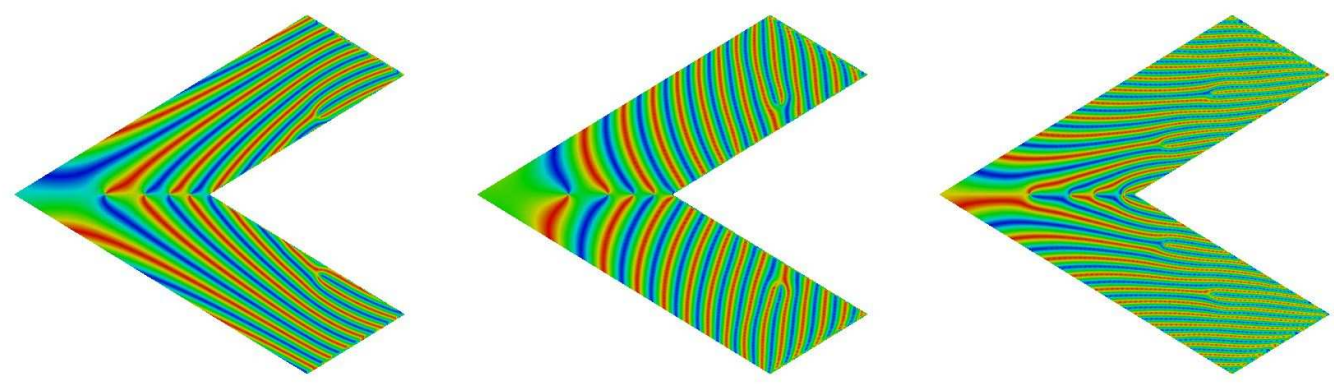

Figure 4: Phases of first eigenvector for gauges $\mathcal{A}, \widetilde{\mathcal{A}}, \widehat{\mathcal{A}}$.

The curve in Figure 5 plots the minimum value obtained from these configurations for any opening. We have also represented on the graph the bottom of the essential spectrum $\Theta_{0} \simeq 0.5901$ and the lower and upper bounds given in (4). Since the numerical estimates for the bottom of the spectrum give an upper-bound of $\mu_{1}(\alpha)$, we are ensured that $\mu_{1}(\alpha)<$ $\Theta_{0}$ for any $\alpha \in\{j \pi / 200, j=1, \ldots, 190\}$. We have gathered in Table 1 the value of $\mu_{1}(\alpha)$ obtained for $\alpha=j \pi / 40$. The comparison between the numbers obtained with $\mathbb{Q}_{9}$ and $\mathbb{Q}_{10^{-}}$ 
approximations provides an accuracy estimation for the computed eigenvalues. Numerical experiments for $\alpha \in[\pi, 6 \pi / 5]$ do not show eigenvalue less than $\Theta_{0}$ with similar meshes as in Figure 1

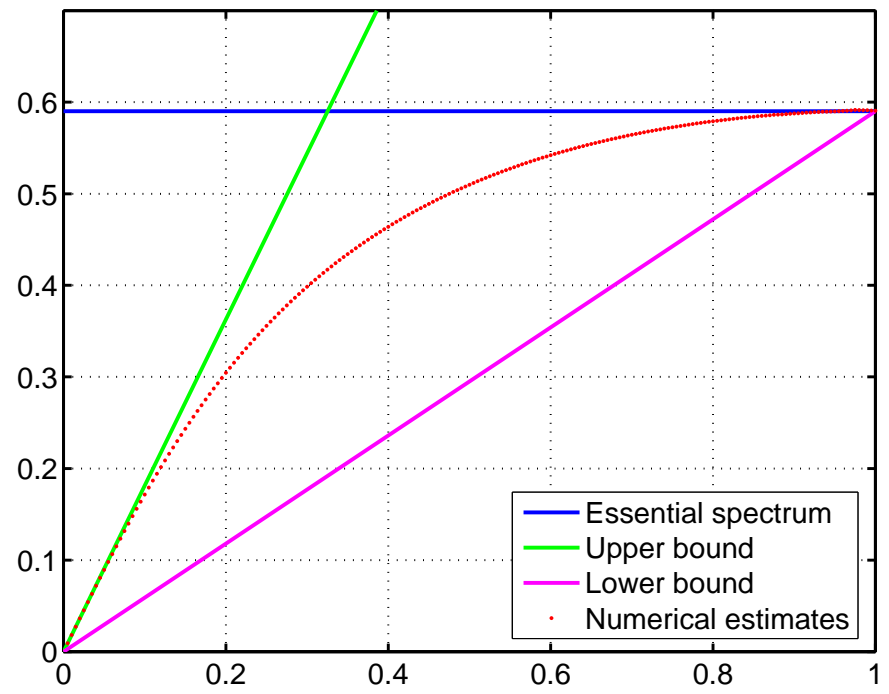

Figure 5: $\mu_{1}(\alpha)$ vs. $\frac{\alpha}{\pi}$ for $\alpha \in[0, \pi]$.

\begin{tabular}{|c|c|c|c|c|c|c|c|c|}
\hline & \multicolumn{2}{|c|}{$\mu_{1}\left(j \frac{\pi}{40}\right)$} & \multicolumn{3}{|c|}{$\mu_{1}\left(j \frac{\pi}{40}\right)$} & \multicolumn{3}{|c|}{$\mu_{1}\left(j \frac{\pi}{40}\right)$} \\
\hline$j$ & $\mathbb{Q}_{9}$ & $\mathbb{Q}_{10}$ & $j$ & $\mathbb{Q}_{9}$ & $\mathbb{Q}_{10}$ & $j$ & $\mathbb{Q}_{9}$ & $\mathbb{Q}_{10}$ \\
\hline 1 & 0.04516 & 0.04516 & 16 & 0.46400 & 0.46400 & 31 & 0.57623 & 0.57614 \\
\hline 2 & 0.08930 & 0.08930 & 17 & 0.47704 & 0.47704 & 32 & 0.57924 & 0.57922 \\
\hline 3 & 0.13160 & 0.13160 & 18 & 0.48898 & 0.48897 & 33 & 0.58193 & 0.58185 \\
\hline 4 & 0.17153 & 0.17153 & 19 & 0.49990 & 0.49990 & 34 & 0.58430 & 0.58415 \\
\hline 5 & 0.20883 & 0.20883 & 20 & 0.50991 & 0.50991 & 35 & 0.58632 & 0.58619 \\
\hline 6 & 0.24339 & 0.24339 & 21 & 0.51907 & 0.51907 & 36 & 0.58819 & 0.58763 \\
\hline 7 & 0.27524 & 0.27524 & 22 & 0.52745 & 0.52745 & 37 & 0.58997 & 0.58904 \\
\hline 8 & 0.30447 & 0.30447 & 23 & 0.53512 & 0.53512 & 38 & 0.59030 & 0.59000 \\
\hline 9 & 0.33123 & 0.33123 & 24 & 0.54213 & 0.54213 & 39 & 0.60130 & 0.59149 \\
\hline 10 & 0.35570 & 0.35570 & 25 & 0.54853 & 0.54852 & 40 & 0.59087 & 0.59064 \\
\hline 11 & 0.37806 & 0.37806 & 26 & 0.55435 & 0.55435 & & & \\
\hline 12 & 0.39848 & 0.39848 & 27 & 0.55965 & 0.55964 & & & \\
\hline 13 & 0.41713 & 0.41713 & 28 & 0.56445 & 0.56443 & & & \\
\hline 14 & 0.43418 & 0.43418 & 29 & 0.56880 & 0.56876 & & & \\
\hline 15 & 0.44976 & 0.44976 & 30 & 0.57272 & 0.57265 & & & \\
\hline
\end{tabular}

Table 1: Numerical values for the bottom of the spectrum. 
Remark 3.3. Considering the results in Figure 5 we conjecture that $\mu_{1}$ is strictly increasing from $(0, \pi]$ onto $\left(0, \Theta_{0}\right]$, equal to $\Theta_{0}$ on $[\pi, 2 \pi]$ and that $\mu_{1}^{\prime}(\pi)=0$. Furthermore, from the results in Figure 3 it appears that there is only one eigenvalue $\mu_{1}(\alpha)$ below the essential spectrum for $\alpha \in(\pi / 5, \pi)$.

\section{Square}

We consider here the Schrödinger operator $P_{h}=-(h \nabla-i \mathcal{A})^{2}$ with Neumann boundary conditions on the model square $\Omega_{\mathrm{sq}}=(-1,1) \times(-1,1)$, and the range 1 to 0.01 for the parameter $h$.

\subsection{Theoretical results}

We denote by $\mathrm{s}_{1}=(-1,-1), \mathrm{s}_{2}=(1,-1), \mathrm{s}_{3}=(1,1), \mathrm{s}_{4}=(-1,1)$ the vertices of $\Omega_{\mathrm{sq}}$. The analysis of the eigenpairs of $P_{h}$ on the square fits in the framework of more general polygonal domains, studied in [10]. We give here a specified version of the results, which takes into account the symmetry properties of the square.

Relying on Remark 3.3. we admit that there is only one eigenvalue $\mu_{1}(\pi / 2)$ below $\Theta_{0}$ for the operator $Q^{\pi / 2}$ on the quarter plane and that $\mu_{1}(\pi / 2)$ is simple. Corresponding to the 4 corners of the square, the first 4 eigenpairs of $P_{h}$ derive from 4 quasi-modes generated by the eigenpair $\left(\mu_{1}(\pi / 2), \Psi_{1}^{\pi / 2}\right)$ on the quarter plane:

Notation 4.1. - Let $\mu_{h, n}$ be the $n$-th eigenvalue of $P_{h}$ counted with multiplicity and $u_{h, n}$ be a normalized eigenfunction associated with $\mu_{h, n}$.

- We introduce the sum $F_{h}$ of the first 4 eigenspaces of $P_{h}$ :

$$
F_{h}=\operatorname{span}\left\{u_{h, 1}, u_{h, 2}, u_{h, 3}, u_{h, 4}\right\} .
$$

- We define the corresponding space $E_{h}$ of quasi-modes

$$
E_{h}=\operatorname{span}\left\{\psi_{h, \mathrm{~s}_{1}}, \psi_{h, \mathrm{~s}_{2}}, \psi_{h, \mathrm{~s}_{3}}, \psi_{h, \mathrm{~s}_{4}}\right\}
$$

generated by the 4 functions $\psi_{h, s_{j}}$ defined as follows: Let $j \in\{1,2,3,4\}$ and $\mathcal{R}_{j}$ be the rotation of opening $(j-1) \pi / 2$. We first define the function $\breve{\psi}_{h, \mathbf{s}_{j}}$ by

$$
\breve{\psi}_{h, \mathbf{s}_{j}}(x)=\frac{1}{\sqrt{h}} \exp \left(\frac{i}{2 h} x \wedge \mathrm{s}_{j}\right) \Psi_{1}^{\pi / 2}\left(\frac{\mathcal{R}_{j}\left(x-\mathrm{s}_{j}\right)}{\sqrt{h}}\right) \quad \text { on } \quad \mathcal{R}_{j}^{-1} G^{\pi / 2}
$$

and set

$$
\psi_{h, \mathbf{s}_{j}}(x)=\chi_{j}(x) \breve{\psi}_{h, \mathbf{s}_{j}}(x) \quad \text { on } \quad \Omega_{\mathrm{sq}} .
$$

Here $\chi_{j}$ is a radial smooth cut-off function with support in the ball $B\left(\mathrm{~s}_{j}, 2\right)$ and equal to 1 in $B\left(\mathrm{~s}_{j}, 2-\delta\right)$ for some positive $\delta$.

The quasi-modes $\psi_{h, \mathrm{~s}_{j}}$ allow to compare the eigenvalues of $P_{h}$ with those of $Q^{\pi / 2}$; the distance between the clusters $E_{h}$ and $F_{h}$ can be quantified as well. The results of [10] applied to the situation of a square give the estimates: 
Proposition 4.2. With Notation 4.1 for any $\varepsilon>0$, there exist $C_{\varepsilon}>0$ such that for $n=1,2,3,4$,

$$
\left|\mu_{h, n}-h \mu_{1}\left(\frac{\pi}{2}\right)\right| \leq C_{\varepsilon} \exp \left(-\frac{2 \sqrt{\Theta_{0}-\mu_{1}\left(\frac{\pi}{2}\right)}-\varepsilon}{\sqrt{h}}\right) .
$$

Furthermore, for any $\varepsilon \geq \delta$ :

$$
d\left(E_{h}, F_{h}\right) \leq C_{\varepsilon} \exp \left(-\frac{2 \sqrt{\Theta_{0}-\mu_{1}\left(\frac{\pi}{2}\right)}-\varepsilon}{\sqrt{h}}\right),
$$

where $d$ is the distance defined by $d\left(E_{h}, F_{h}\right)=\left\|\Pi_{E_{h}}-\Pi_{F_{h}} \Pi_{E_{h}}\right\|_{\mathcal{H}}$, with $\Pi_{E_{h}}$ and $\Pi_{F_{h}}$ the orthogonal projections onto $E_{h}$ and $F_{h}$ respectively.

Consequently the eigenvectors associated with the smallest four eigenvalues of $P_{h}$ are exponentially close to a linear combination of the four quasi-modes $\psi_{h, s_{j}}, j=1,2,3,4$. Numerical experiments show that these combinations are not trivial. Furthermore, this theorem also proves that the smallest four eigenvalues of $P_{h}$ form a cluster exponentially close to $h \mu_{1}(\pi / 2)$. Numerical experiments bring more information about the behavior of these eigenvalues, and display fine interactions. Moreover, although no theoretical results are available for eigenvalues of rank larger than 5 (except the fact that they cannot converge below $\Theta_{0} \simeq 0.59$ ), we will see that they also organize into clusters of 4 .

In the following, when representing eigenmodes, we show their moduli and, most often, their phases. The phase is computed according to the formula $\arcsin (\operatorname{Im}(z) /|z|)$.

\subsection{Dependency on $h$ of the first eigenfunction}

Formula (8) exhibits a two-scale structure for the quasi-modes: a corner layer at scale $\sqrt{h}$ coming from the dilatation $\Psi_{1}^{\pi / 2}(\cdot / \sqrt{h})$, and an oscillatory term at scale $h$ due to $\mathrm{e}^{\frac{i}{2 h} x \wedge s}$. Relying on Proposition 4.2 the same holds for the functions in the eigenspace $E_{h}$. Consequently, especially because of harsh oscillations, there is a difficult issue of approximating correctly the eigenfunctions of $P_{h}$ for small values of $h$.

We present as a conclusion of this paper in Section 7 a systematic investigation of errors when discretizing our problem on the square thanks to h-extensions with bilinear elements, or to p-extensions with coarse meshes of 1 to 64 elements. In this section, we choose each time an optimal combination mesh-degree to display eigenmodes.

To compute the first eigenfunction for $h=0.1,0.08,0.06,0.04,0.02,0.01$, we keep the polynomial approximation fixed to $\mathbb{Q}_{10}$ and a $8 \times 8$ mesh. Figure 6 gives the modulus of the first eigenfunction and Figure 7 its phase. As expected, we observe that the modulus is more and more concentrated in the corners and phase has sharper oscillations when $h$ decreases. 

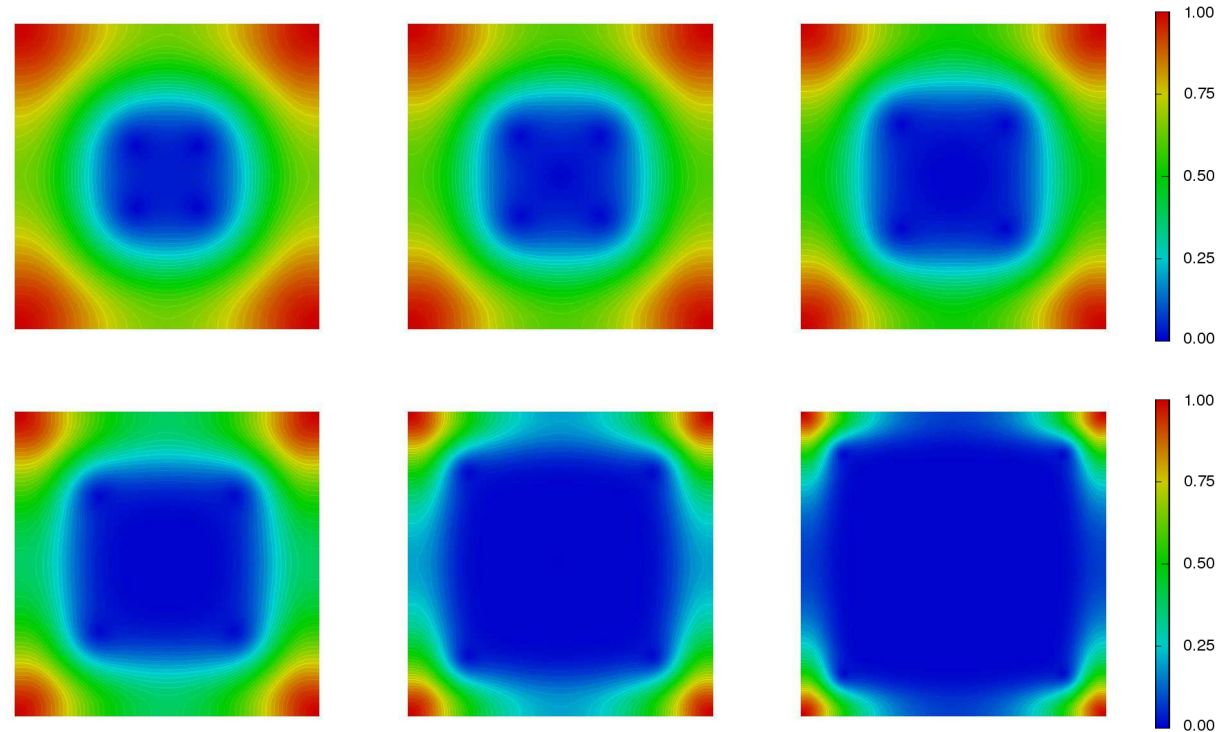

Figure 6: Moduli of the first eigenfunction, $h=0.1,0.08,0.06,0.04,0.02,0.01$.
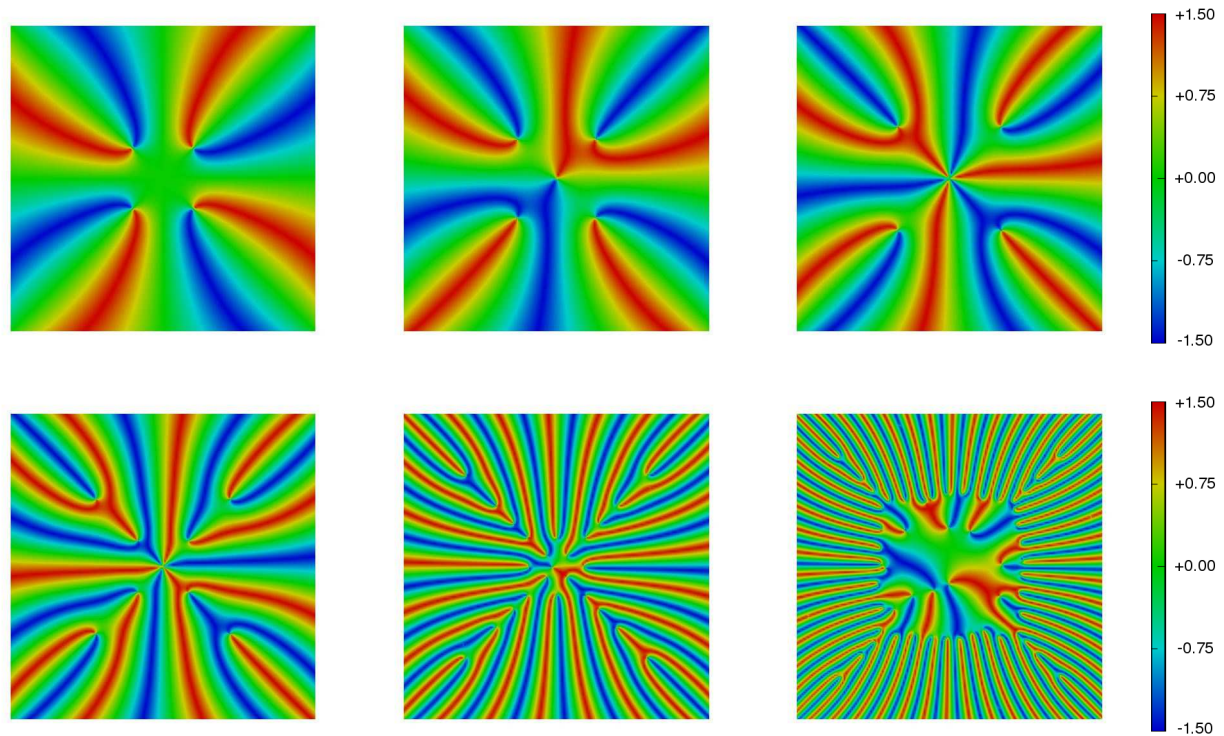

Figure 7: Phases of the first eigenfunction, $h=0.1,0.08,0.06,0.04,0.02,0.01$.

\subsection{Dependency on the rank of eigenfunctions for a given value of $h$}

In Figures 8 we keep $h=0.02$ fixed and compute the eigenfunctions associated with the smallest eight eigenvalues of $P_{h}$. We observe that the eigenvectors associated with the smallest four eigenvalues are localized in the four corners as predicted by Proposition 4.2 . and that, moreover, each one is present in all the four corners, as can be predicted by symme- 
try arguments. There is no theoretical results for the next eigenpairs, but the computations show a localization of the eigenvectors along the edges of the square.

The full portrait (modulus and phase) of the first 32 modes can be found in Appendix A.
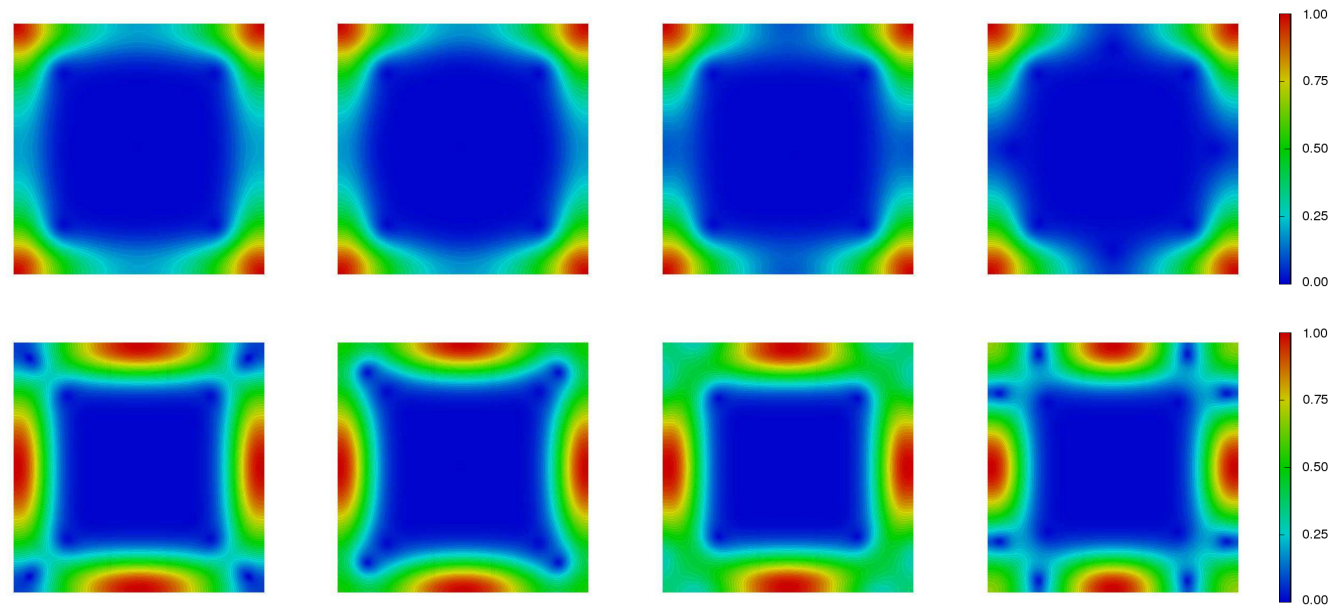

Figure 8: Moduli of the first 8 eigenfunctions, $h=0.02$.

\subsection{Tunneling effect}

The tunneling effect refers to the interaction between symmetric potential wells, see [21] for instance. In our situation, the tunneling effect applies to corners of the same aperture. If present, this effect is an interaction of eigenvalues inside the same cluster, possibly stronger than the convergence of the whole cluster to its asymptotic limit. It could be formally evaluated by investigating the eigenpairs of the Galerkin projection on the space of quasimodes $\breve{\psi}_{h, \mathrm{~s}_{j}}$.

Here, we simply compute, not only the first 4 , but the first 12 eigenvalues, with a $\mathbb{Q}_{10}$ approximation on uniform meshes of 4 to 64 elements, according to the value of $1 / h$, ranging from 1 to 90, with step 0.5. We present in Figure 9 the graph of these first 12 eigenvalues divided by $h$, vs. $h^{-1}$. We observe that the eigenvalues interlace inside clusters of four. The first cluster, converging to the value $\mu_{1}(\pi / 2) \simeq 0.5099$, is contained in an exponential tube (materialized in the figure by the dashed curves of equation $h^{-1} \mapsto 0.5099 \pm 0.6 \exp \left(-0.5665 h^{-1 / 2}\right)$ as a numerical representation of the asymptotics (10)). The further clusters remain higher than $\Theta_{0} \simeq 0.59$.

We note that, since $P_{h}$ is self-adjoint and its coefficients depend analytically on $h$, its eigenvalues can be organized to display an analytic dependence on $h$ in any interval disjoint from 0 . By a simple automatic postprocessing of the results, we follow eigenvalues as families depending smoothly on $h$.

The multiple crossings between eigenvalues are corroborated by a closer look at the eigenvectors: Tracking the symmetry properties of eigenvectors, it becomes obvious that the crossings really occur. These oscillations are due to the magnetic field, and do not exist in presence of an electric field alone. 

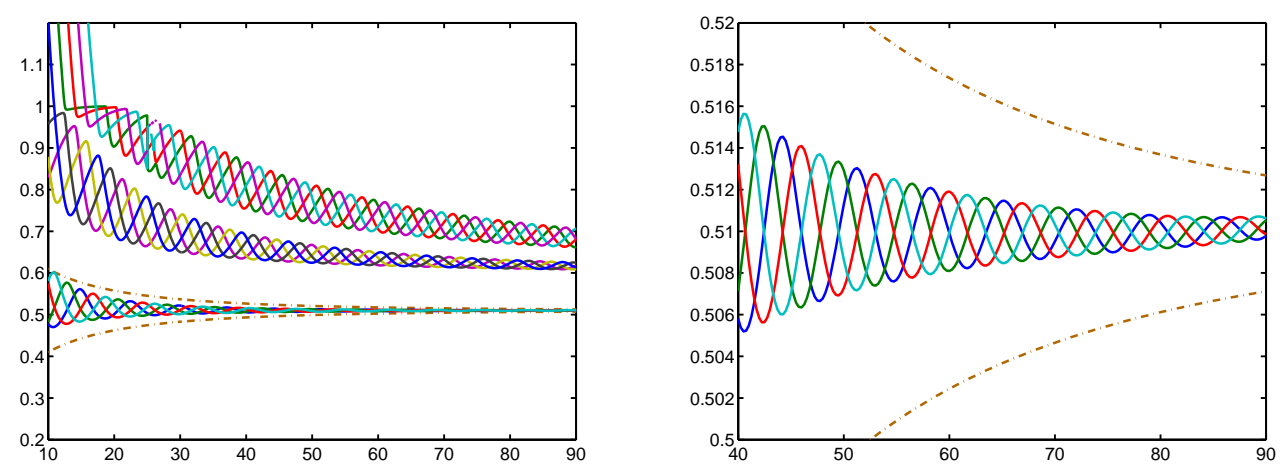

Figure 9: $h^{-1} \mu_{h, n}$ vs. $h^{-1}, n=1, \ldots, 12$ in $\Omega_{\mathrm{sq}}$ (left). Zoom to the first cluster (right).

\section{Other polygons}

Let now $\Omega$ denote a general polygon with straight edges. The behavior of the lowest eigenvalues of the Neumann realization $P_{h}$ of the operator $-(h \nabla-i \mathcal{A})^{2}$ on $\Omega$ as $h$ tends to 0 has, in a certain sense, the same features as previously.

Let $\Sigma$ be the set of the vertices s of $\Omega$, and $\alpha_{\mathrm{s}}$ be the opening of $\Omega$ at the vertex s. As already seen in the case of the square, the spectrum of $P_{h}$ is in close relation with the spectra of the model operators $Q^{\alpha_{\mathrm{s}}}$ for s describing the set of corners $\Sigma$.

\subsection{Theoretical results}

Let us suppose for sake of simplicity that, for any vertex s, the model operator $Q^{\alpha_{\mathrm{s}}}$ has at most one eigenvalue $\mu_{1}\left(\alpha_{\mathrm{s}}\right)$. This is the case for the examples we propose. From previous computations, see Remark 3.3, it is enough that the openings $\alpha_{\mathrm{s}}$ are greater than $\pi / 5$. See [10] for the general case.

Let $\Sigma_{1}$ be the set of vertices s such that $\mu_{1}\left(\alpha_{\mathrm{s}}\right)<\Theta_{0}$. From Remark 3.3 again, $\Sigma_{1}$ coincides with the set of convex vertices of $\Omega$.

Notation 5.1. - Let $\mu_{h, n}$ be the $n$-th eigenvalue of $P_{h}$ counted with multiplicity.

- Let $\lambda_{n}$ be the $n$-th element of the set $\left\{\mu_{1}\left(\alpha_{\mathrm{s}}\right), \mathrm{s} \in \Sigma_{1}\right\}$.

- Let $\rho$ be the minimum distance between two corners of $\Omega$.

Theorem 5.2. With Notation 5.1 for any $\varepsilon>0$, there exists $C_{\varepsilon}$ such that

$$
\left|\mu_{h, n}-h \lambda_{n}\right| \leq C_{\varepsilon} \exp \left(-\frac{1}{\sqrt{h}}\left(\rho \sqrt{\Theta_{0}-\lambda_{n}}-\varepsilon\right)\right), \quad \forall n \leq N:=\#\left(\Sigma_{1}\right) .
$$

Thus, according to repetitions of the same value $\lambda$ in $\left\{\lambda_{1}, \ldots, \lambda_{N}\right\}$, the corresponding eigenvalues $\mu_{h, n}$ are gathered into clusters, exponentially close to the same value $h \lambda$. It is proved in [10] that the corresponding eigenvectors are exponentially close to linear combinations of quasi-modes: Quasi-modes $\psi_{h, s}$ are defined by translation, rotation, scaling, and 
cut-off from the eigenvectors $\Psi_{1}^{\alpha_{\mathrm{s}}}$ for any $\mathrm{s} \in \Sigma_{1}$ like in Notation 4.1 for the square,

$$
\psi_{h, \mathrm{~s}}(x)=\chi_{\mathrm{s}}(x) \frac{1}{\sqrt{h}} \exp \left(\frac{i}{2 h} x \wedge \mathrm{s}\right) \Psi_{1}^{\alpha_{\mathrm{s}}}\left(\frac{\mathcal{R}_{\mathrm{s}}(x-\mathrm{s})}{\sqrt{h}}\right) .
$$

Notation 5.3. - Using Notation 5.1 we denote by $\left\{\Lambda_{1}<\cdots<\Lambda_{M}\right\}$ the set of distinct values in $\left\{\lambda_{1}, \ldots, \lambda_{N}\right\}$.

- For any $m \leq M$, we define the $m$-th cluster of eigenspaces of $P_{h}$ by

$$
F_{h, m}=\operatorname{span}\left\{u_{h, n} \mid \forall n \text { such that } \lambda_{n}=\Lambda_{m}\right\},
$$

and the corresponding cluster $F_{h, m}$ of quasi-modes

$$
E_{h, m}=\operatorname{span}\left\{\psi_{h, \mathbf{s}} \mid \forall \mathrm{s} \in \Sigma_{1} \text { such that } \mu_{1}\left(\alpha_{\mathbf{s}}\right)=\Lambda_{m}\right\} .
$$

Theorem 5.4. For any $\varepsilon \geq \delta$, with $\delta$ depending on the cut-off functions $\chi_{\mathrm{s}}$, there exists $C_{\varepsilon}>0$ such that for any $m \leq M$,

$$
d\left(E_{h, m}, F_{h, m}\right) \leq C_{\varepsilon} \exp \left(-\frac{1}{\sqrt{h}}\left(\rho \sqrt{\Theta_{0}-\Lambda_{m}}-\varepsilon\right)\right) .
$$

\subsection{Rhombus and Trapezoid}

We consider two examples of convex quadrilateral domains, a rhombus $\Omega_{\mathrm{rh}}$ with two pairs of distinct openings, and a trapezoid $\Omega_{\mathrm{tr}}$ without symmetry with two openings equal.

The corners of the rhombus $\Omega_{\mathrm{rh}}$ are $\mathrm{s}_{1}=(-\sqrt{2} / 2,0), \mathrm{s}_{2}=(0,-\sqrt{2}), \mathrm{s}_{3}=(\sqrt{2} / 2,0)$, $\mathrm{s}_{4}=(0, \sqrt{2})$. As illustrated in Figure 10, for $h=0.02$ the first two eigenvectors are localized in the smallest openings, whereas the third and the fourth one are localized in the largest openings. Because of symmetry, these eigenvectors are localized in two corners and not in one only.
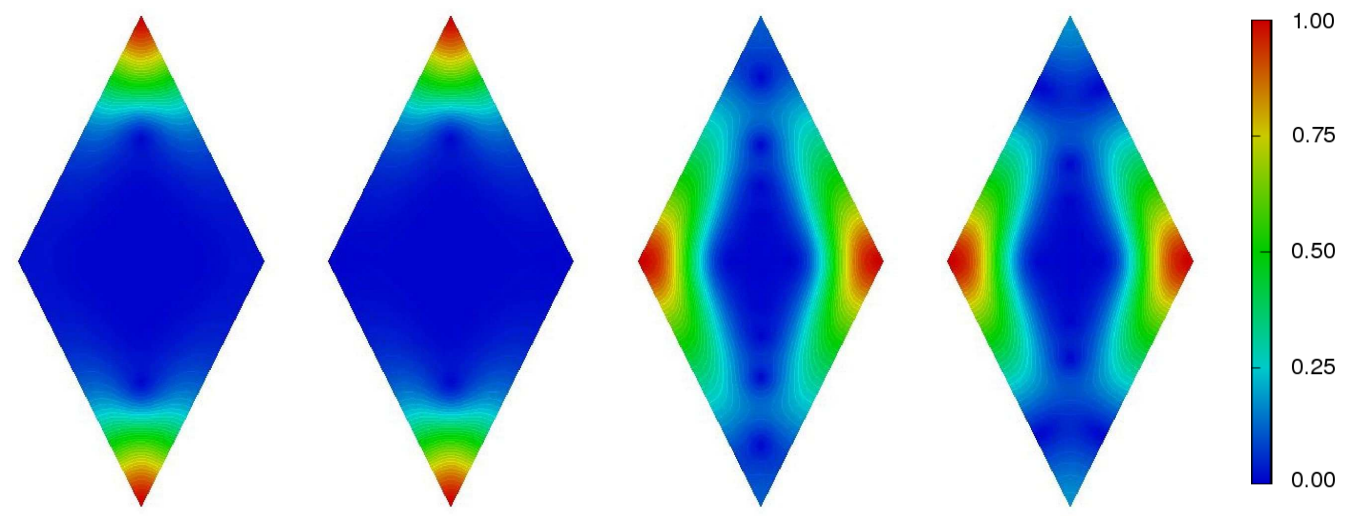

Figure 10: Moduli of eigenvectors 1 to 4 in $\Omega_{\mathrm{rh}}$ for $h=0.02$.

The corners of the trapezoid $\Omega_{\mathrm{tr}}$ are $\mathrm{s}_{1}=(-1,-1), \mathrm{s}_{2}=(1,-1), \mathrm{s}_{3}=(1,0), \mathrm{s}_{4}=$ $(-1,1)$. Thus the openings at $s_{1}$ and $s_{2}$ are equal to $\pi / 2$. We show in Figure 11 the 
first four eigenvectors for $h=0.02$. As expected, the corners are visited according to increasing magnitude. An interesting difference from the symmetric case is the localization of eigenvectors 2 and 3 in corners $s_{1}$ and $s_{2}$ with quite different coefficients. We have noticed that the concentration in one corner only is stronger as $h$ gets smaller. The pictures of moduli in $\log 10$ scale (bottom) give another insight on the support of eigenvectors.
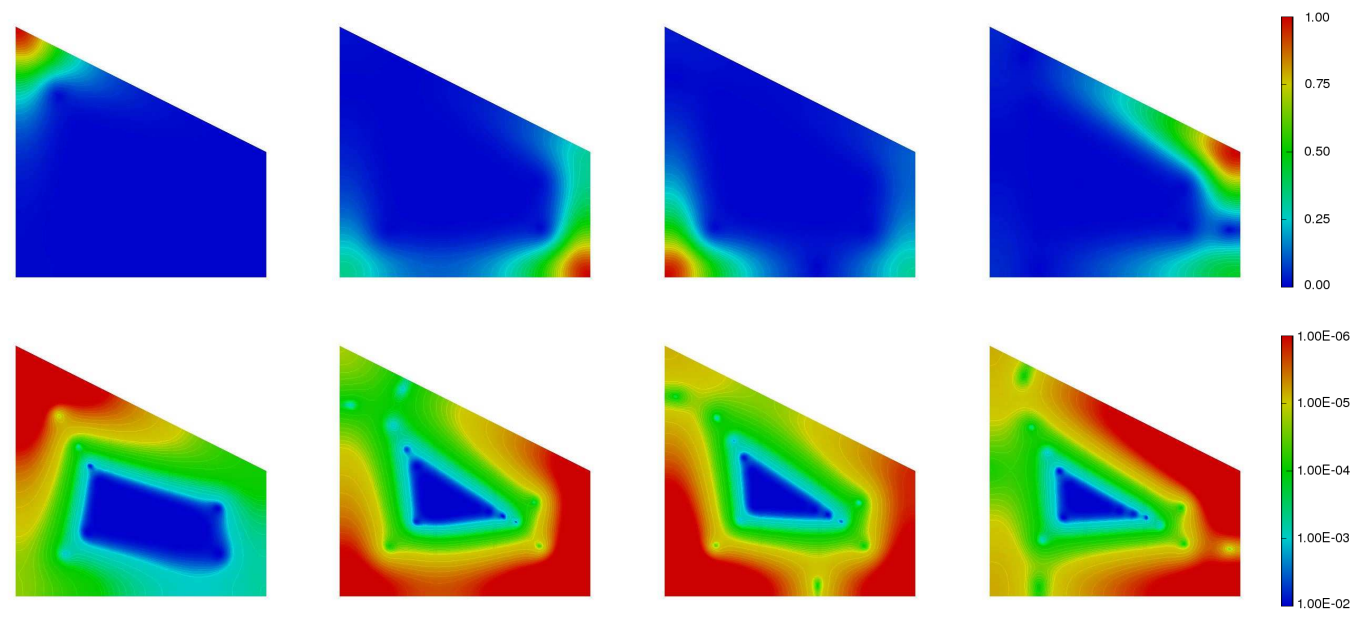

Figure 11: Moduli of eigenvectors 1 to 4 in $\Omega_{\mathrm{tr}}$ for $h=0.02$.

Natural color scale (top) and logarithmic color scale (bottom)

The plots of $h^{-1} \mu_{h, n}$ vs. $h^{-1}$ display two convergent two-element clusters for the rhombus (note the values of $\mu_{1}(\alpha)$ estimated by the method in 43 for the two different openings: 0.395 and 0.565 ), and three distinct limits for the trapezoid (note: $\mu_{1}(\alpha) \simeq 0.434,0.510$ and 0.554). Eigenvalues interlace much less in the trapezoid, because of the absence of symmetry.
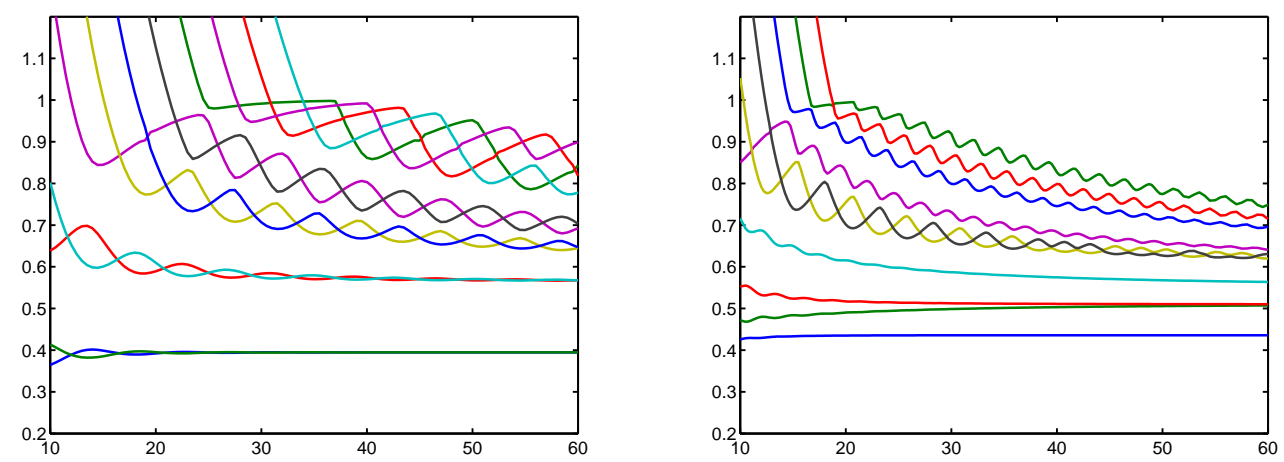

Figure 12: $h^{-1} \mu_{h, n}$ vs. $h^{-1}$ for $\Omega_{\mathrm{rh}}$ (left) and $\Omega_{\mathrm{tr}}$ (right). 


\subsection{L-shape}

The L-shaped domain $\Omega_{\mathrm{L}}$ has six corners: $\mathrm{s}_{1}=(0,0), \mathrm{s}_{2}=(2,0), \mathrm{s}_{3}=(2,1), \mathrm{s}_{4}=$ $(1,1), \mathrm{s}_{5}=(1,2), \mathrm{s}_{6}=(0,2)$. Thus it has 5 corners of same opening $\pi / 2$ and one non-convex corner. The big five element cluster around $\mu_{1}(\pi / 2)$ splits in fact in three sub-clusters of 2, 1 and 2 elements, respectively, see Figure 14
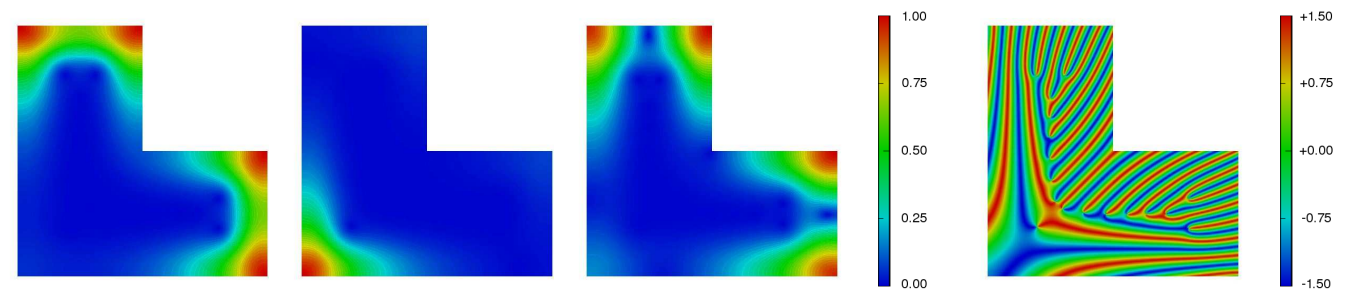

Figure 13: Moduli of eigenvectors 1, 3 and 5 in $\Omega_{\mathrm{L}}$, phase of eigenvector 1 .

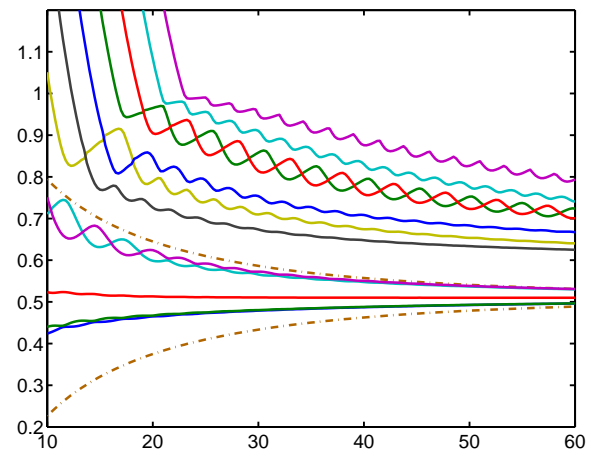

Figure 14: $h^{-1} \mu_{h, n}$ vs. $h^{-1}$ for the L-shape $\Omega_{\mathrm{L}}$.

\section{Curvilinear polygonal domains}

If $\Omega$ is a curvilinear polygon, as proved in [10], we still have convergence of the eigenpairs of $P_{h}$ towards those of $\oplus_{\mathbf{s} \in \Sigma_{1}} Q^{\alpha_{\mathrm{s}}}$, but instead of being exponential, the convergence has the rate $\sqrt{h}$. Nevertheless, clustering and tunnelling are still present if the domain is symmetric, as shown on the curved square $\Omega_{\text {curv }}$ below. The opening of the angles of $\Omega_{\text {curv }}$ is equal to $0.650 \pi$, corresponding to $\mu_{1}(\alpha)=0.554$. A geometrical interpolation of degree 4 has been used for the design of the $8 \times 8$ mesh.

From Figure 16, the slower convergence and weaker concentration of eigenvalues inside their cluster are visible, when compared to the case of the square (see Figure 9 ). 

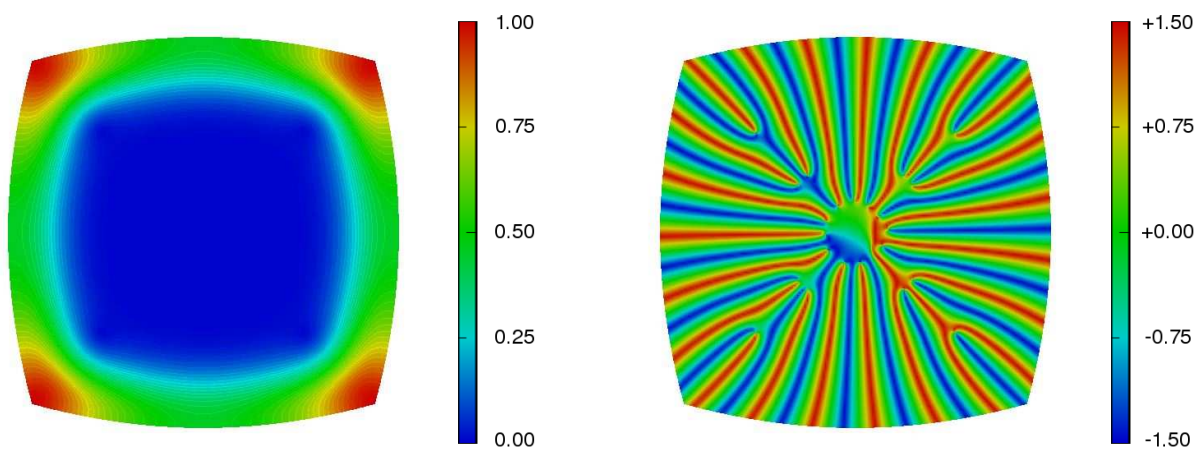

Figure 15: First eigenvector on the curved square $\Omega_{\text {curv }}$ (modulus and phase).
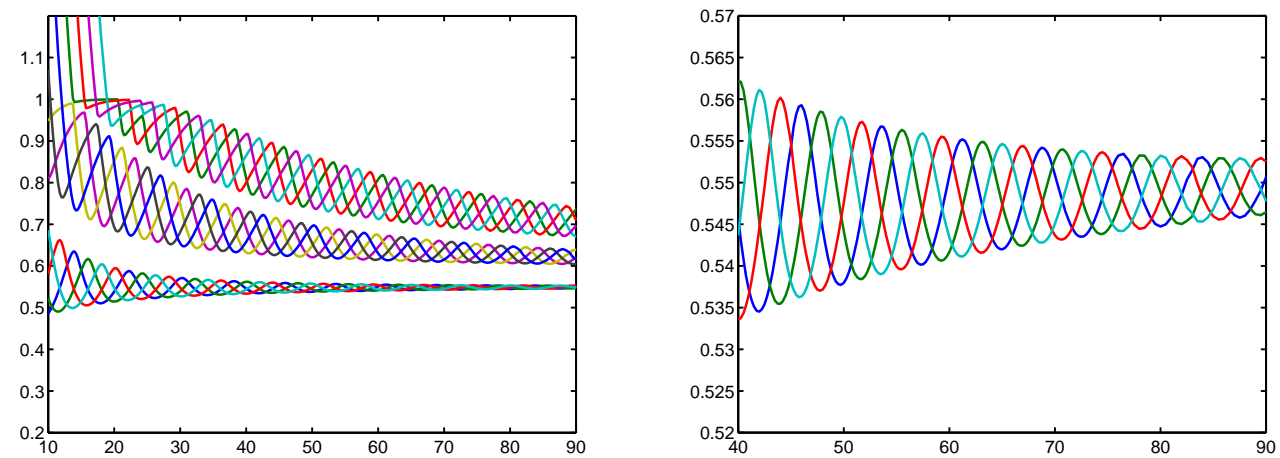

Figure 16: $h^{-1} \mu_{h, n}$ vs. $h^{-1}, n=1, \ldots, 12$, in the curved square $\Omega_{\text {curv }}$.

\section{7 h-extension vs. p-extension}

We now compare in a systematic way the performances of the h-extension (i.e. keep the polynomial degree fixed and refine the mesh) with those of the p-extension (i.e. keep the mesh fixed and increase the polynomial degree). All numerical experiments are carried with the standard square $\Omega_{\text {sq }}$ centered at $(0,0)$ with side length 2 .

In 7.1 we keep the number of degrees of freedom (Dof) equal to 1600 and compare the dependency on the small parameter $h$ of eigenvalues computed with different combinations of meshes and degrees. In $\$ 7.2$ and $\$ 7.3$ the parameter $h$ is set to 0.02 and show errors for h- and p-extensions, respectively.

\subsection{Several combinations mesh-degree}

We compute the first eight eigenvalues of $P_{h}$ on the square $\Omega_{\mathrm{sq}}$ for $h^{-1}=10$ to 60 by step 0.5 with four different combinations of 1600 Dof: $\mathbb{Q}_{1}$ in a $40 \times 40$ mesh, $\mathbb{Q}_{2}$ in a $20 \times 20$ mesh, $\mathbb{Q}_{5}$ in a $8 \times 8$ mesh, and, finally, $\mathbb{Q}_{20}$ in a $2 \times 2$ mesh. We plot in Figures 17 
the first eight discrete eigenvalues divided by $h$, vs. $h^{-1}$, and according to their smooth dependency in $h$ (like for Figure 9. And, like in Figure 9 for the same reason, we plot in dashed lines the exponential tube $h^{-1} \mapsto 0.5099 \pm 0.6 \exp \left(-0.5665 h^{-1 / 2}\right)$. We recall that we expect the first four eigenvalue cluster to concentrate inside this tube.
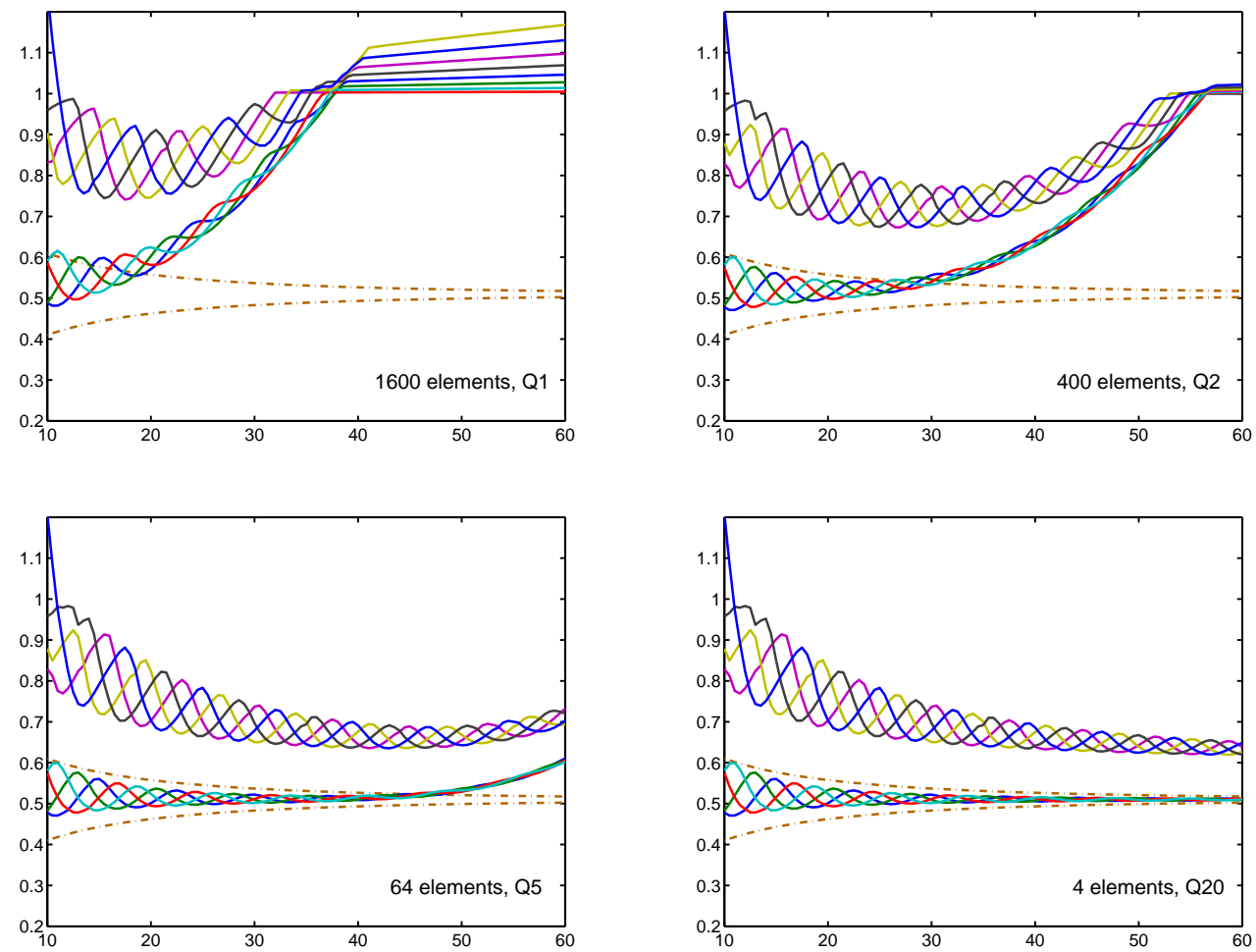

Figure 17: $h^{-1} \mu_{h, n}$ vs. $h^{-1}$ for 1600 Dof

Besides the clearly visible better performance of high degree, two features are noticeable: (i) While they are below 1, the eight eigenvalues still gather into two clusters and interlace with each other, and (ii) when eigenvalues get higher than 1 , they stick to this value and do not oscillate any more.

\section{2 h-extension with degree 1 and 2}

The semi-classical parameter $h$ is fixed to 0.02 . The reference value is taken to 0.50726621 for $h^{-1} \mu_{h, 1}$, and is obtained with $\mathbb{Q}_{12}$-approximation on the $8 \times 8$ mesh.

From Figure 18, we observe a preasymptotic convergence to 1, followed by the asymptotic convergence towards $h^{-1} \mu_{h, 1}$. The preasymptotic convergence appears to be faster. A closer look at the log-log plots of Figure 19 shows that the convergence rates are similar: If $\ell$ denotes the mesh size, the rates are approximately $\ell^{2}$ and $\ell^{4}$ for $\mathbb{Q}_{1}$ and $\mathbb{Q}_{2}$, respectively, but the errors behave like $C \ell^{2}$ and $C \ell^{4}$ with a much larger $C$ for the asymptotic convergence than for the preasymptotic one. 


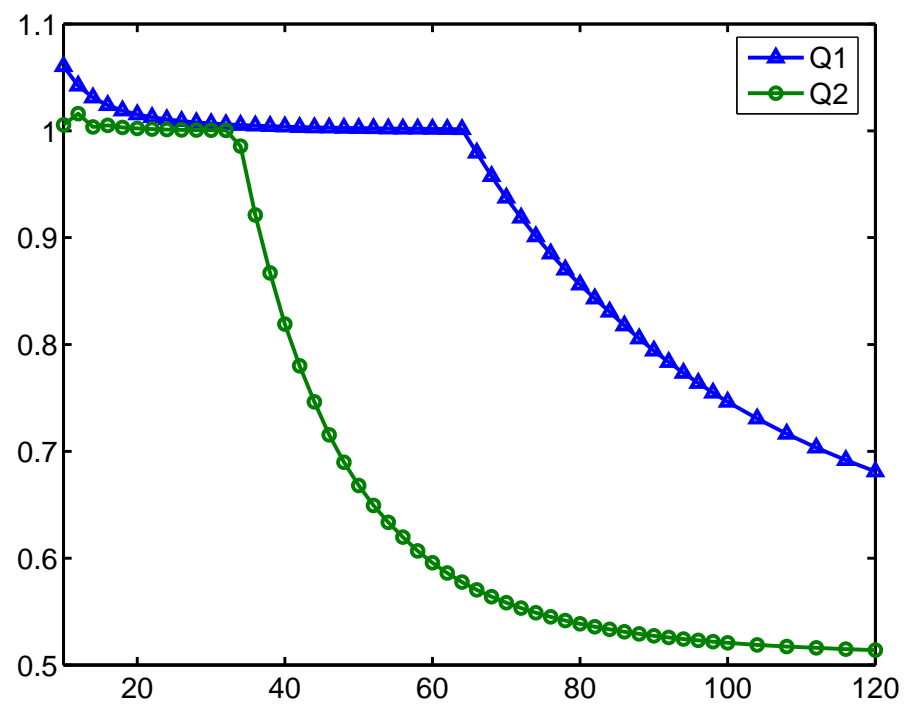

Figure 18: $h^{-1} \mu_{h, 1}$ vs. number of Dof per side for $\mathbb{Q}_{1}$ and $\mathbb{Q}_{2}$-approximation.
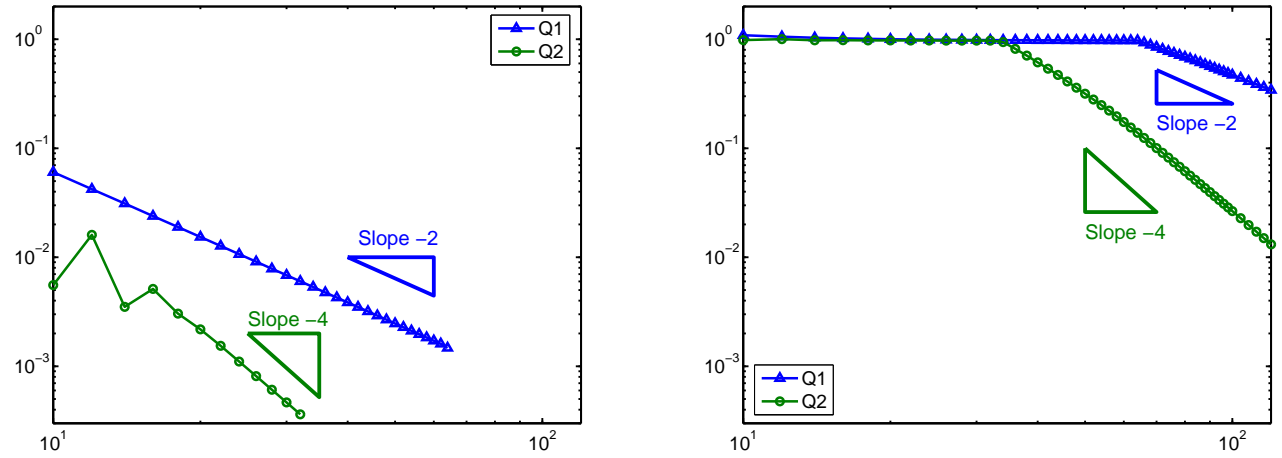

Figure 19: Relative errors for first eigenvalue, vs. number of Dof per side. Errors wrt preasymptotic value 1 (left), and to exact value 0.50726621 (right)

The structure of theses results evokes a possible crossing between two very different modes. We display the portrait (modulus and phase) of the first eight eigenvectors computed with a $\mathbb{Q}_{1}$-approximation on a $63 \times 63$ mesh (i.e., the last one before the bifurcation point of the $\mathbb{Q}_{1}$ curve, cf. Figure 18.

It is clear that modes 1, 2, 3, 5 and 8 are of different nature, and that modes 4,6 and 7 are somewhat closer to "true" modes 5-8, see Figure 8 These less oscillating modes, especially 1-3, look like the first Landau modes

$$
\left(\mathrm{X}_{1}, \mathrm{X}_{2}\right) \longmapsto\left(\mathrm{X}_{1}+i \mathrm{X}_{2}\right)^{n} \exp \left(-\frac{1}{4}\left(\mathrm{X}_{1}^{2}+\mathrm{X}_{2}^{2}\right)\right),
$$

for $n=0,1,2$ and the scaling $\mathbf{X}=\frac{x}{\sqrt{h}}$. The Landau modes are a basis of the (infinite 

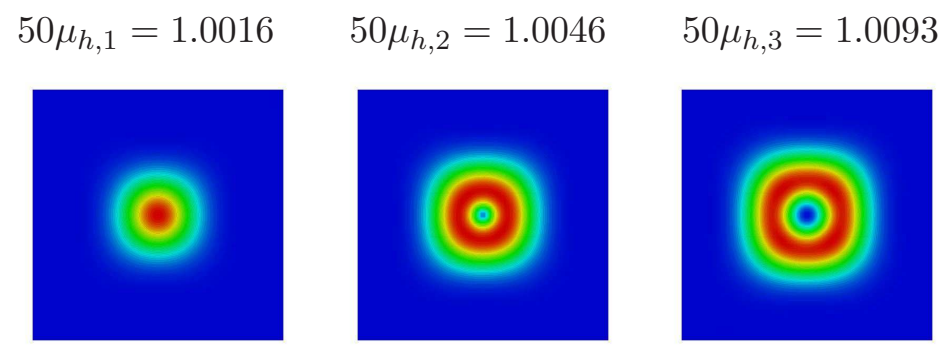

$50 \mu_{h, 4}=1.0130$
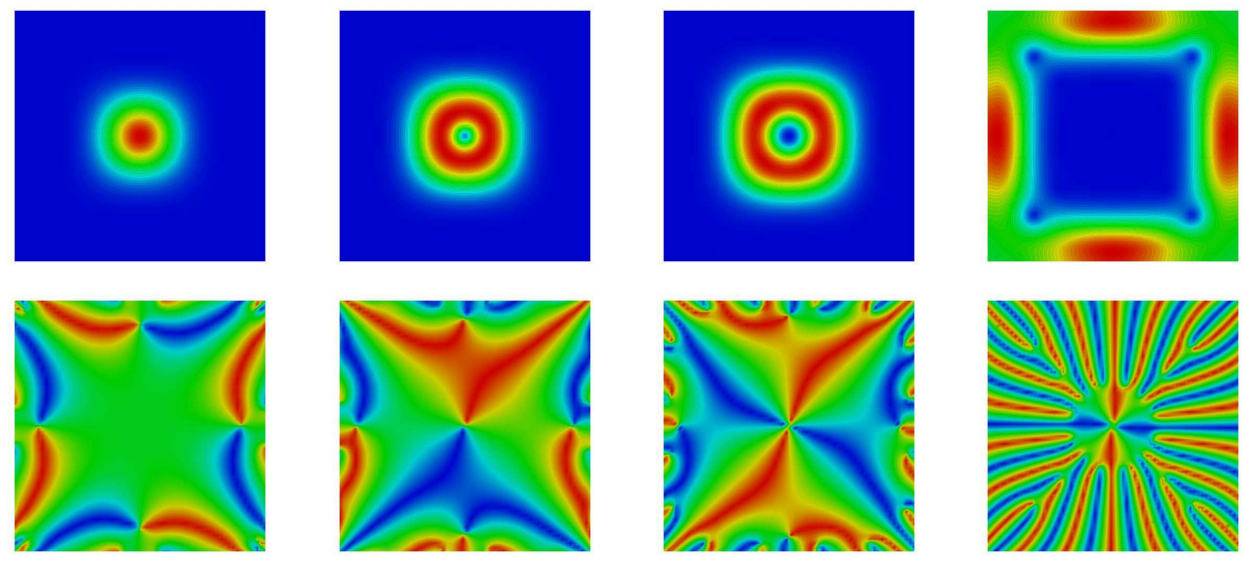

$50 \mu_{h, 5}=1.0154$

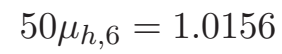

$50 \mu_{h, 7}=1.0219$
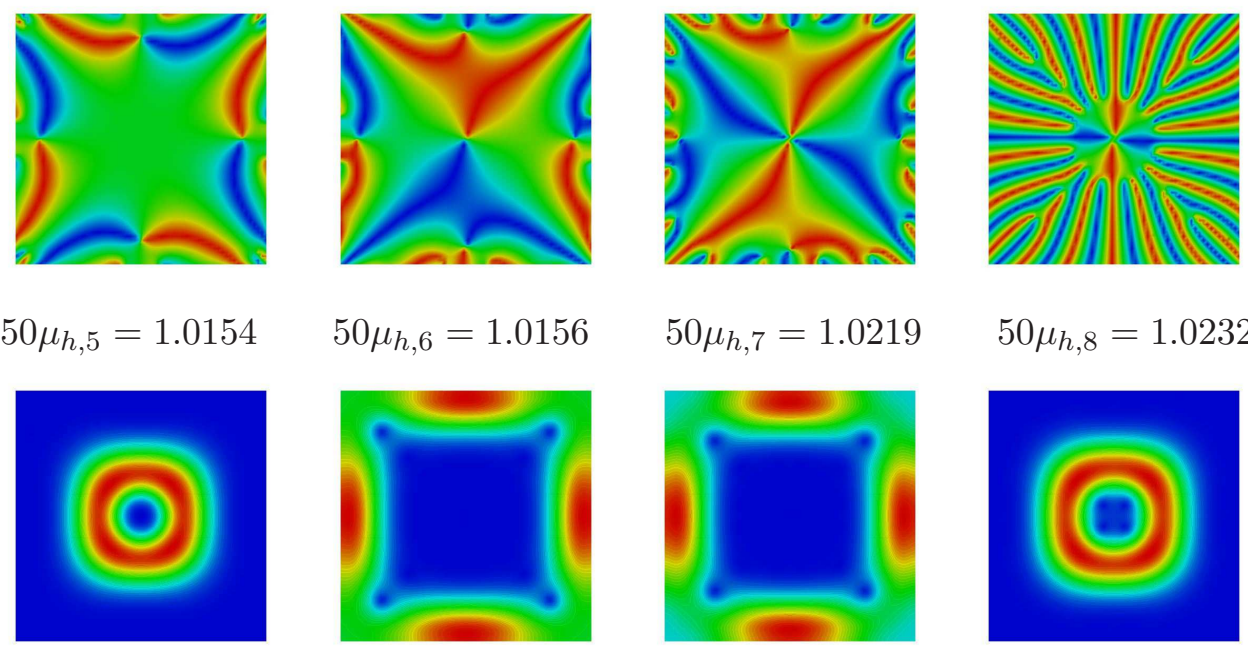

$50 \mu_{h, 8}=1.0232$
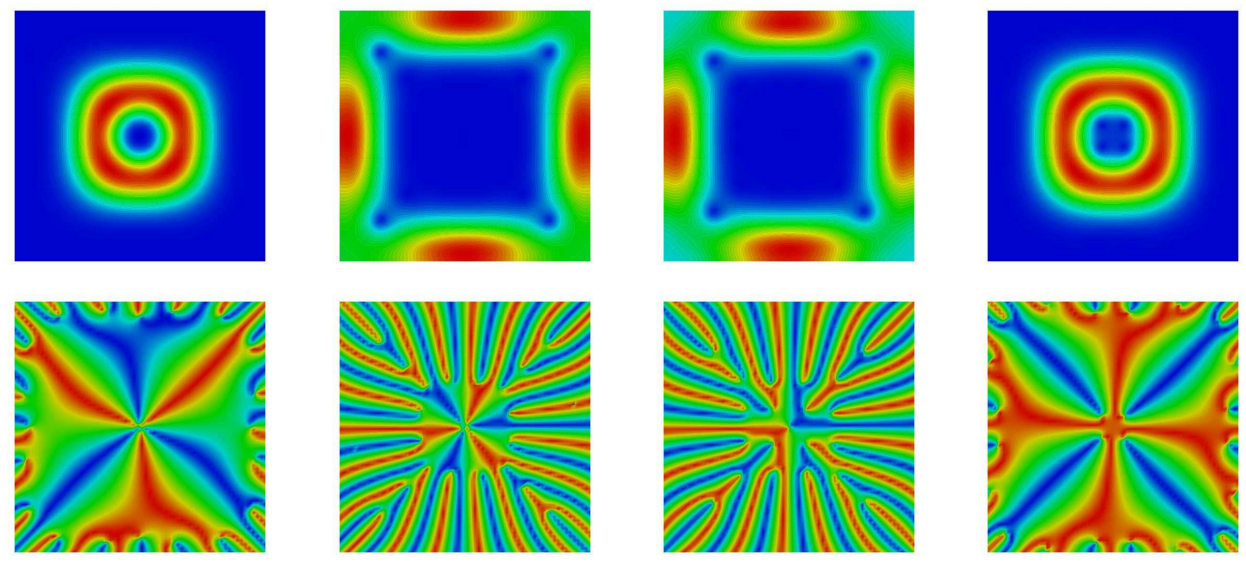

Figure 20: Modes 1 to $8, h=0.02, \mathbb{Q}_{1}$-approximation on $63 \times 63$ mesh. Moduli (top) and phases (bottom)

dimensional) eigenspace of the operator $-(\nabla-i \mathcal{A})^{2}$ in $\mathbb{R}^{2}$, for the lowest Landau level, that is, 1 .

Examining the sequence of the first 32 modes computed with the $\mathbb{Q}_{10}$-approximation on the $8 \times 8$ mesh, we can see that some of them, especially 32, 31 and 30, also look like the first three Landau modes (cf. Appendix A).

From the $63 \times 63$ mesh to the $64 \times 64$ mesh, we do observe crossings between modes: For instance mode 4 becomes mode 1 . Besides, the structure of oscillating modes 4, 6 ad 7 produced with the $63 \times 63$ mesh is very close to that of exact modes. 


\section{3 p-extension with coarse meshes}

As in the previous section, the semi-classical parameter $h$ is fixed to 0.02 and the reference value is the same.

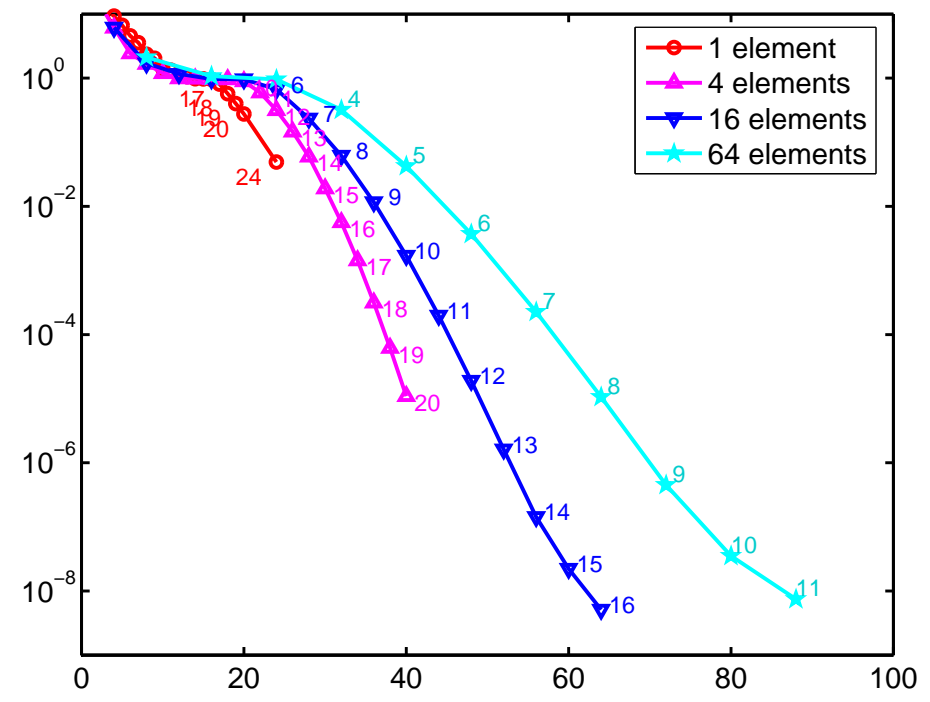

Figure 21: Relative errors for first eigenvalue, vs. number of Dof per side.

Semi-logarithmic scale from $10^{-9}$ to 10 for errors. Integers mark polynomial degree.

We plot on separate curves the errors for each mesh, letting the degree vary. We still notice a locking region, where $h^{-1} \mu_{h, 1}$ converges to 1 (which also corresponds to a relative error $\simeq 1$ ). This region expands with the number of elements of the mesh. But, as a result, with the same number of Dof the p-extension is far more precise than the h-extension.

\section{Conclusion}

Even with a size ratio equal to 100 between the domain $\Omega$ and the semi-classical parameter $h$ (this is the case for $\Omega_{\mathrm{sq}}$ with $h=0.02$ ), the computation of the eigenpairs of $P_{h}$ is a numerical challenge for two reasons: $(i)$ the double scale for the first eigenvectors, inducing oscillations of wave length $\mathcal{O}(h)$ (ii) the presence of different asymptotic modes, possibly less oscillatory, like the Landau modes.

In fact, depending on the position of the domain with respect to the gauge center, the preasymptotic convergence will have quite different features: For a small enough $h$ fixed like in the previous section, decreasing the mesh size with a low degree approximation provides a preasymptotic concentration of the first mode around the gauge center. This phenomenon has been observed in [25], too. Thus it is very useful to know the asymptotic

\footnotetext{
${ }^{2}$ The somewhat odd fact that the first mode is increasingly oscillating as $h \rightarrow 0$ has some similarity with the situation of sensitive shells, see [12].
} 
behavior of eigenmodes before trying to compute them. This prevents to believe that the first mode is approached as soon as a convergence appears.

From a more theoretical point of view, this rather simple, but very rich, example proves the importance and the role of the global constant $L_{h, n}^{\ell, p}$ in estimates (1) and (2). The capability of approximating the first mode of the continuous problem is not sufficient for a precise computation of its first eigenpair, cf. Figure 20 In other words, we do not have the strict analogue of Céa lemma for eigenpair approximation. Nevertheless, the obvious better performances of $\mathrm{p}$-extension over h-extension have some connection to the approximability of oscillatory functions by high degree polynomials, better than by piecewise affine or quadratic functions.

As a last remark, we notice the absence of influence of corner singularities for this problem: $(i)$ eigenmodes are mainly supported outside non-convex corners and (ii) the effect of singularities at convex corners will be felt after the oscillations are resolved (beyond a relative error of $10^{-6}$ in Figure 21. The main role played by these oscillations, and the fact that they spread everywhere in the domain makes it useless to refine meshes near corners. On the contrary, uniform meshes have provided the most precise results regarding fine interactions between corners (the tunnelling effect).

\section{References}

[1] Ainsworth, M. Discrete dispersion relation for $h p$-version finite element approximation at high wave number. SIAM J. Numer. Anal. 42, 2 (2004), 553-575 (electronic).

[2] Almog, Y. The onset of superconductivity in long rectangles. European J. Appl. Math. 14, 3 (2003), 257-277.

[3] Alouges, F., And Bonnaillie-NoËL, V. Numerical computations of fundamental eigenstates for the Schrödinger operator under constant magnetic field. To appear in Numerical Methods for PDE (2005).

[4] BABUŠKA, I., AND Osborn, J. E. Finite element-Galerkin approximation of the eigenvalues and eigenvectors of self-adjoint problems. Math. Comp., 186 (1989), 275297.

[5] BAbUŠKA, I., And Osborn, J. E. Eigenvalue problems, vol. 2. Handbook of numerical analysis, 1991.

[6] Bauman, P., Phillips, D., And Tang, Q. Stable nucleation for the GinzburgLandau system with an applied magnetic field. Arch. Rational Mech. Anal. 142, 1 (1998), 1-43.

[7] Bernoff, A., And Sternberg, P. Onset of superconductivity in decreasing fields for general domains. J. Math. Phys. 39, 3 (1998), 1272-1284.

[8] Bonnaillie, V. On the fundamental state energy for a Schrödinger operator with magnetic field in domains with corners. Asymptot. Anal. 41, 3-4 (2005), 215-258. 
[9] Bonnaillie-NoËL, V. A posteriori error estimator for the eigenvalue problem associated to the Schrödinger operator with magnetic field. Numer. Math. 99, 2 (2004), 325-348.

[10] Bonnaillie-NoËL, V., AND DAuge, M. Asymptotics for the low-lying eigenstates of the Schrödinger operator with magnetic field near corners. Preprint IRMAR 05-27 (2005).

[11] Chatelin, F. Spectral Approximations of Linear operators. Academic Press, New York, 1983.

[12] Dauge, M., Faou, E., And Yosibash, Z. Plates and shells: Asymptotic expansions and hierarchical models. In Encyclodepia of Computational Mechanics, Vol. 1, E. Stein, R. de Borst, and T. J. Hughes, Eds. Wiley, 2004, pp. 199-236.

[13] De Gennes, P. G. Superconductivity in metals and Alloys. Addison Wesley, 1989.

[14] Del Pino, M., Felmer, P. L., And Sternberg, P. Boundary concentration for eigenvalue problems related to the onset of superconductivity. Comm. Math. Phys. 210, 2 (2000), 413-446.

[15] Fournais, S., And HelfFer, B. Accurate eigenvalue estimates for the magnetic Neumann Laplacian. To appear in Annales Inst. Fourier (2005).

[16] Fournais, S., And HelfFER, B. Energy asymptotics for type II superconductors. Calc. Var. 24, 3 (2005), 341-376.

[17] Fournais, S., And HelfFer, B. On the third critical field in Ginzburg-Landau theory. Preprint Orsay 2005-37 (2005).

[18] Helffer, B., And Mohamed, A. Semiclassical analysis for the ground state energy of a Schrödinger operator with magnetic wells. J. Funct. Anal. 138, 1 (1996), 40-81.

[19] HelfFer, B., And Morame, A. Magnetic bottles in connection with superconductivity. J. Funct. Anal. 185, 2 (2001), 604-680.

[20] Helffer, B., And Pan, X.-B. Upper critical field and location of surface nucleation of superconductivity. Ann. Inst. H. Poincaré Anal. Non Linéaire 20, 1 (2003), 145181.

[21] Helffer, B., And Sjöstrand, J. Multiple wells in the semiclassical limit. I. Comm. Partial Differential Equations 9, 4 (1984), 337-408.

[22] IhlenburG, F., AND BABUŠKA, I. Finite element solution of the Helmholtz equation with high wave number. I. The $h$-version of the FEM. Comput. Math. Appl. 30, 9 (1995), 9-37.

[23] Ihlenburg, F., And BABUŠKa, I. Finite element solution of the Helmholtz equation with high wave number. II. The $h-p$ version of the FEM. SIAM J. Numer. Anal. 34, 1 (1997), 315-358.

[24] Jadallah, H. T. The onset of superconductivity in a domain with a corner. J. Math. Phys. 42, 9 (2001), 4101-4121. 
[25] JANANE, R. Études numériques du spectre d'un opérateur de Schrödinger avec champ magnétique constant. Thèse de doctorat, Université de Nantes, 2005.

[26] LU, K., AND PAN, X.-B. Estimates of the upper critical field for the Ginzburg-Landau equations of superconductivity. Phys. D 127, 1-2 (1999), 73-104.

[27] Martin, D. The finite element library Mélina. http://perso.univ-rennes1.fr/daniel.martin/melina (2005).

[28] Pan, X.-B. Upper critical field for superconductors with edges and corners. Calc. Var. Partial Differential Equations 14, 4 (2002), 447-482.

[29] Tinkham, M. Introduction to superconductivity. McGraw Hill, 1996.

\section{Authors' address}

IRMAR (UMR CNRS 6625)

Université de Rennes 1,

Campus de Beaulieu

35042 RENNES Cedex

FRANCE

Corresponding author

Monique Dauge, monique.dauge@univ-rennes1.fr 


\section{Appendix A: The first 32 eigenpairs in the square for $h=0.02$.}

Computed in $\Omega_{\text {sq }}$ with the $\mathbb{Q}_{10}$-approximation on the $8 \times 8$ mesh.

We give, for each computed eigenmode, its rank $n$, the value of $h^{-1} \mu_{h, n}$, the modulus and the phase of a normalized eigenvector.

1: 0.50727
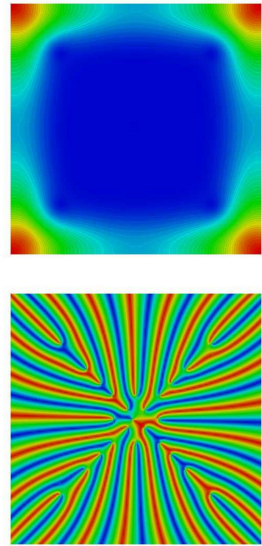

5: 0.62449
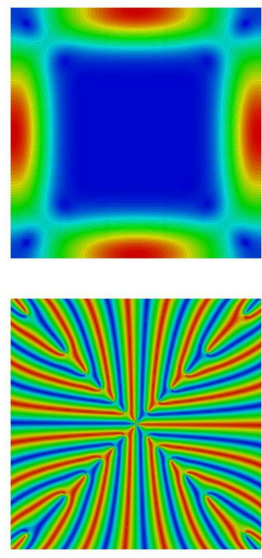

2: 0.50863
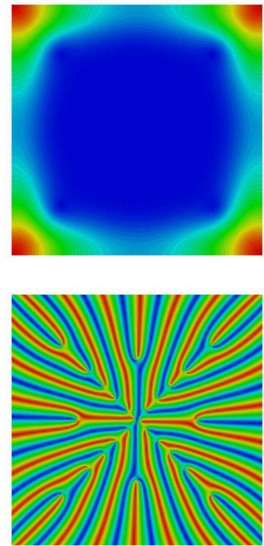

6: 0.63889
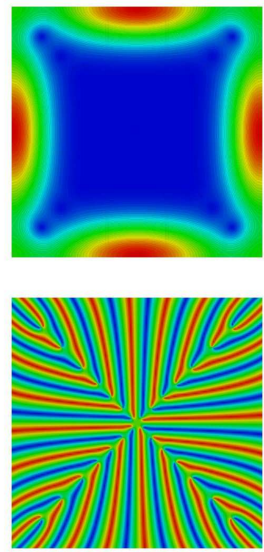

3: 0.51129
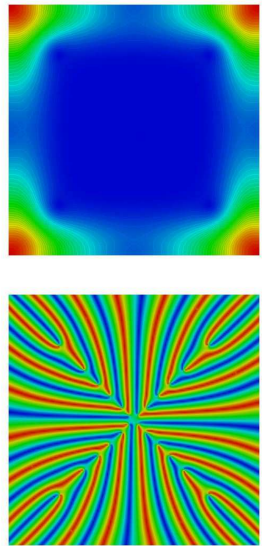

7: 0.64291
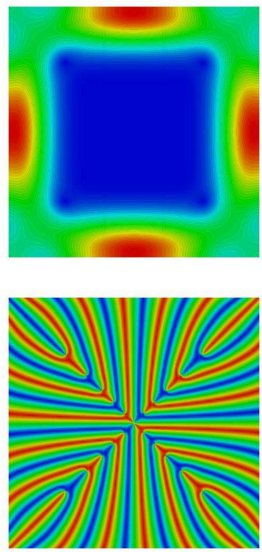

4: 0.51293
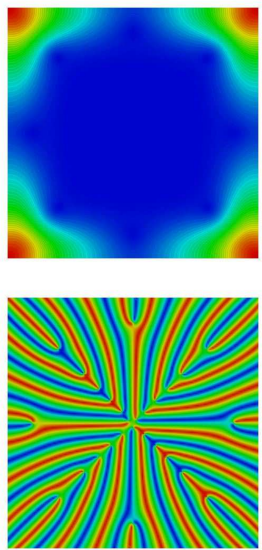

8: 0.66752
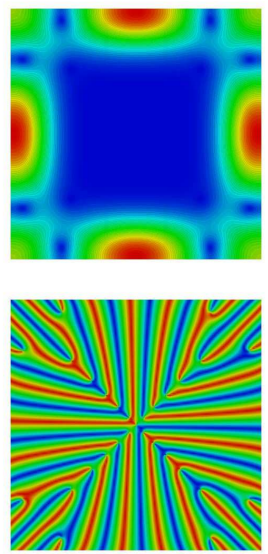

Figure 22: Modes 1 to 8, Modulus (top) and phase (bottom). 

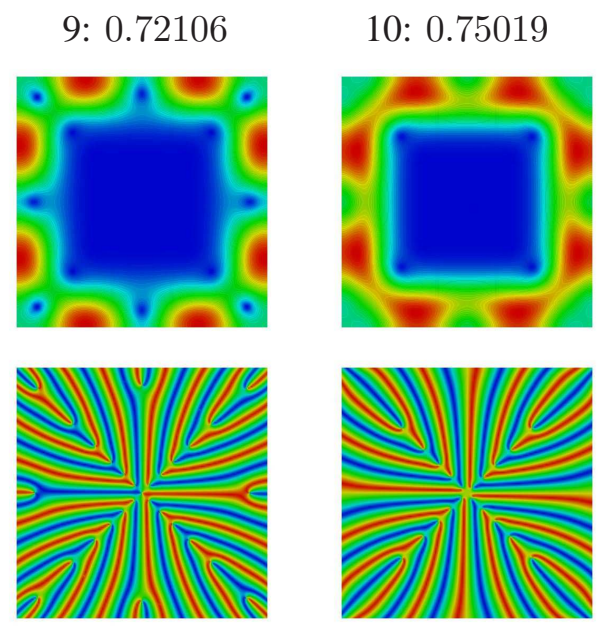

11: 0.77995

12: 0.80027
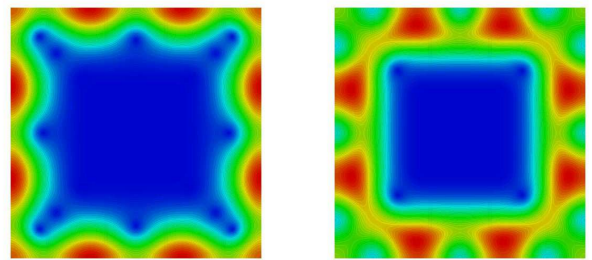

13: 0.86274
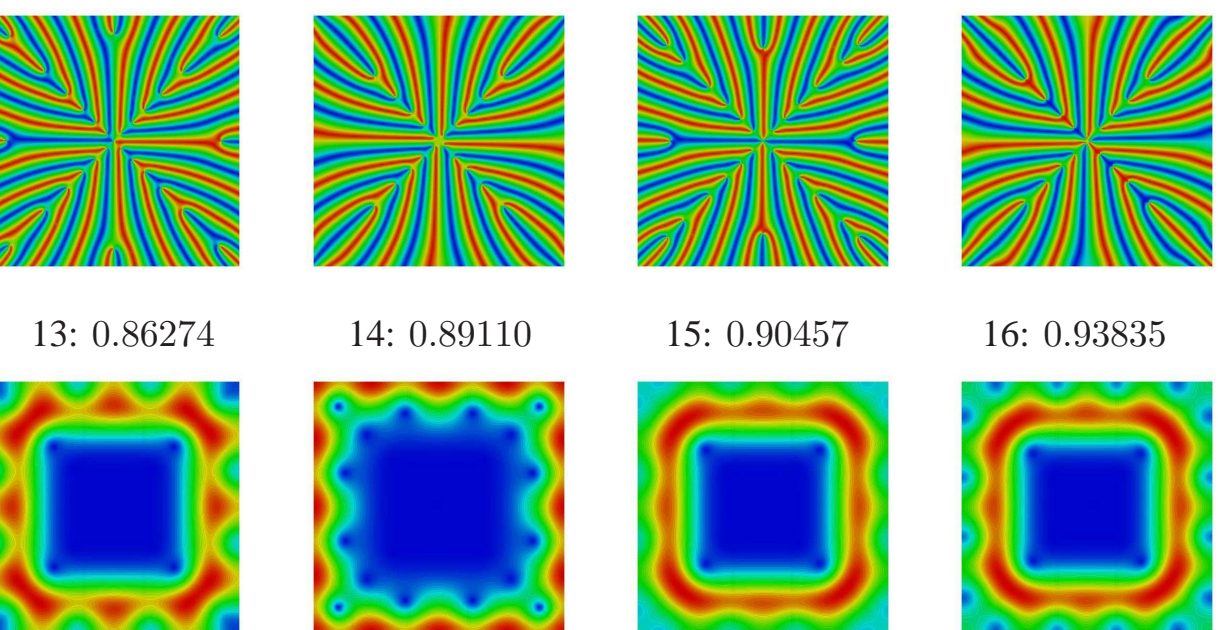

14: 0.89110

15: 0.90457

16: 0.93835
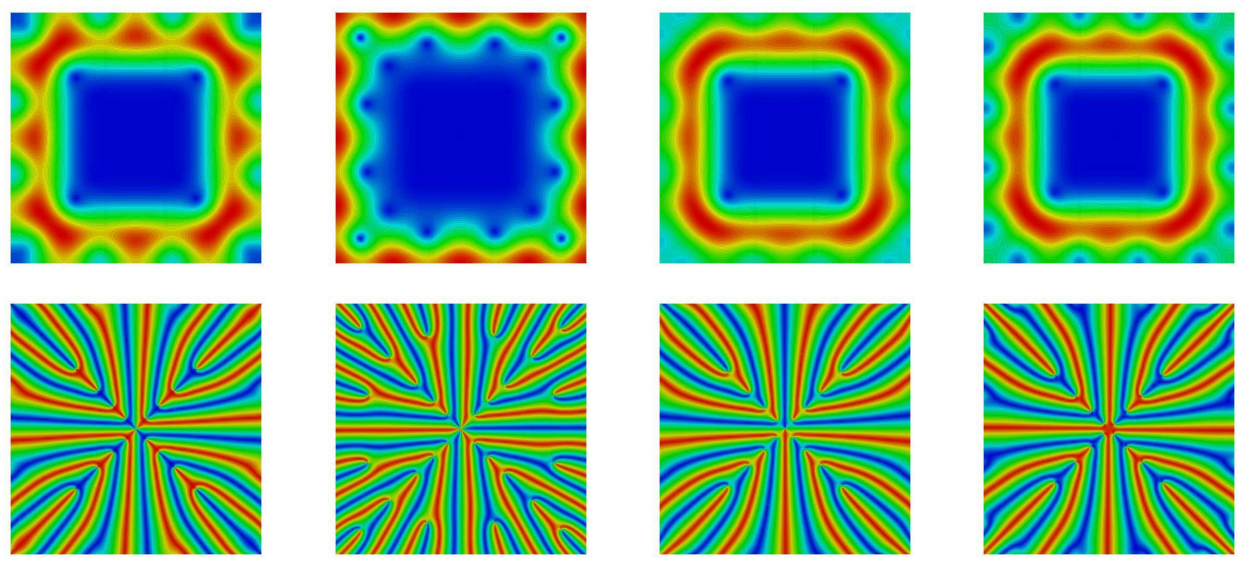

17: 0.96564

18: 0.98089

19: 0.99023

20: 0.99516
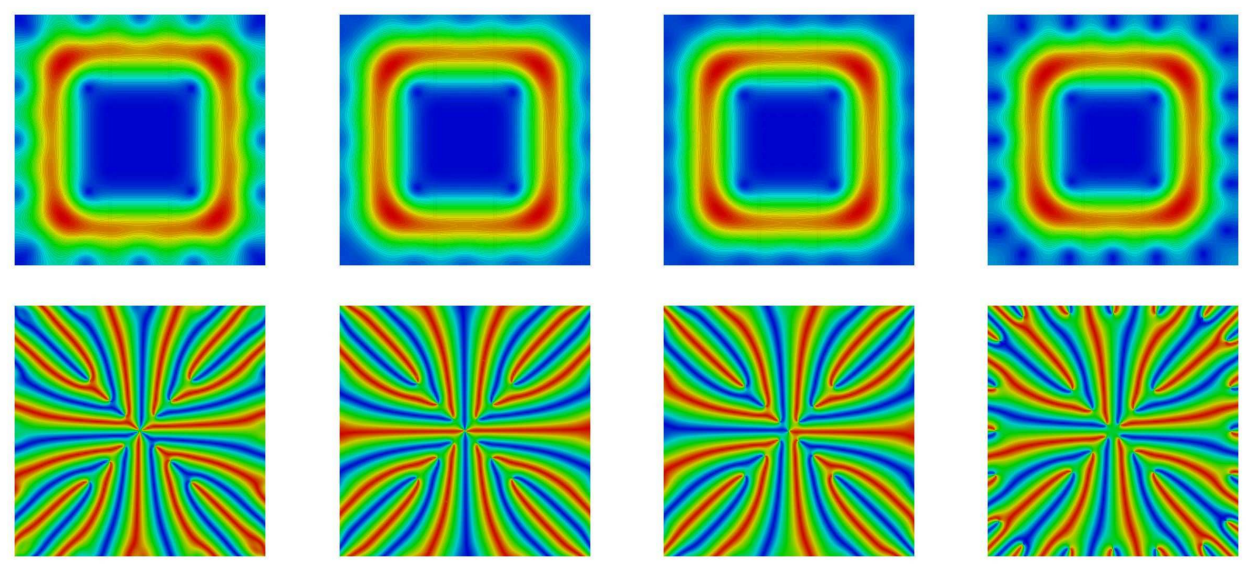

Figure 23: Modes 9 to 20, Modulus (top) and phase (bottom). 

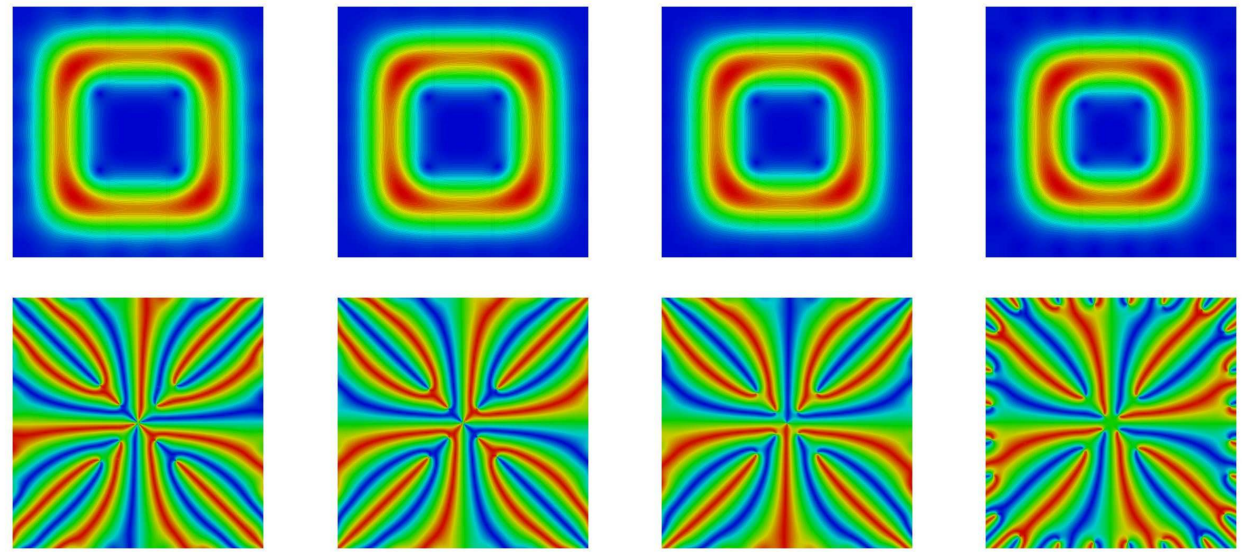

25: 0.99997317614

26: 0.99999318548

27: 0.99999852222

28: 0.99999972716
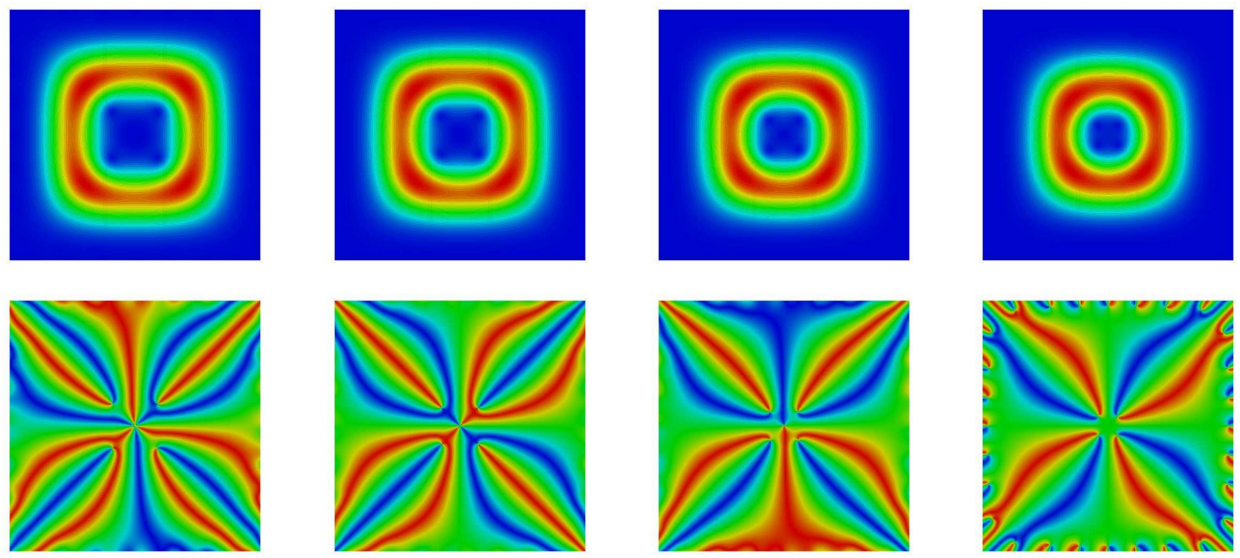

29: 0.99999996227

30: 0.99999999595

31: 0.99999999971

32: 0.99999999999
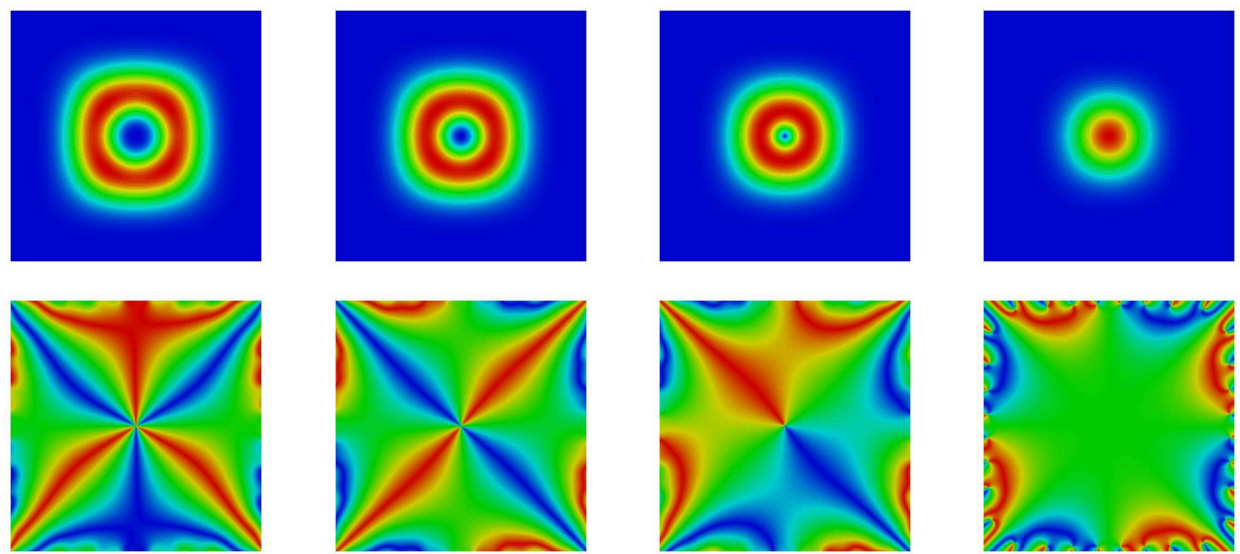

Figure 24: Modes 21 to 32, Modulus (top) and phase (bottom). 


\section{Appendix B: Table of $\mu_{1}(\alpha)$ vs. $\alpha$}

In the next two tables, in each column the integer number $j$ determines $\alpha$ by $\alpha=j \pi / 1000$, the real number besides is $\mu_{1}(\alpha)$.

\begin{tabular}{|c|c|c|c|c|c|c|c|c|c|}
\hline 1 & 0018138 & 41 & 0.073586 & 81 & 0.14141 & 121 & 0.20305 & 161 & 0.25773 \\
\hline 2 & 0.0036275 & 42 & 075342 & 2 & 0.14303 & 122 & 0.2045 & 62 & 025 \\
\hline 3 & 0.0054411 & 43 & 0.077096 & 3 & 0.14465 & 123 & 0.20595 & 63 & \\
\hline 4 & 0.0072544 & 44 & 0.078848 & 4 & 0.146 & 124 & 20739 & 164 & 26155 \\
\hline 5 & 0.0090675 & 45 & 0.0805 & 5 & 0.147 & 125 & 20883 & 165 & 26282 \\
\hline 6 & 0.01088 & 46 & 0.0823 & 6 & 0.14 & 126 & 77 & 166 & 26408 \\
\hline 7 & 0.012693 & 47 & & 87 & 0.15 & 127 & & 167 & 26534 \\
\hline 8 & 0.01 & 48 & & 88 & 0. & 128 & & 168 & 26659 \\
\hline 9 & 0.01 & 49 & & 9 & 0. & 129 & & 169 & \\
\hline 10 & 0. & 50 & & 0 & & 130 & & 170 & \\
\hline 11 & 0. & 51 & & 91 & & 131 & & 171 & \\
\hline 12 & 0. & 52 & & 92 & & 132 & & 172 & 56 \\
\hline 13 & & 53 & & 9. & & 133 & & 173 & \\
\hline 14 & & 54 & & 94 & & 134 & & 17 & 01 \\
\hline 15 & $0 .($ & 55 & & 95 & & 135 & & 17 & 524 \\
\hline 16 & 0.0 & 56 & & 9 & & 136 & & 17 & 645 \\
\hline 17 & 0.03 & 57 & & 9 & 0.1 & 137 & & 17 & 767 \\
\hline 18 & 0.03 & 58 & & 9 & 0.1 & 138 & & 17 & 0.27888 \\
\hline 19 & 0.03 & 59 & & 9 & 0.16 & 139 & 52 & 179 & 0.28009 \\
\hline 20 & 0.03 & 60 & & 100 & $0.1^{\prime}$ & 140 & & 180 & 0.28129 \\
\hline 21 & 0.03 & 61 & & & 0.1 & 141 & & 18 & 0.28248 \\
\hline 22 & 0.039 & 62 & & & 0.17 & 142 & & 18 & 0.28368 \\
\hline 23 & 0.041576 & 63 & 0 & & 0.176 & 143 & 99 & 18 & 0.28487 \\
\hline 24 & 0.043 & 64 & & & 0.17 & 144 & 535 & 184 & 0.28605 \\
\hline 25 & 0.045164 & 65 & 0 & & 0.17 & 145 & 2367 & 185 & 0.28723 \\
\hline 26 & 0.04 & 66 & 2 & & 0.18 & 146 & 305 & 186 & .28841 \\
\hline 27 & 0.048 & 67 & 0 & 107 & 0.18 & 147 & 23939 & 187 & .28958 \\
\hline 28 & 0.050533 & 68 & 97 & 108 & 0.18 & 148 & 0.24073 & 188 & 29075 \\
\hline 29 & 0.052319 & 69 & 54 & 109 & 0.18527 & 149 & 0.24206 & 189 & .29192 \\
\hline 30 & 0.054103 & 70 & 31 & 5 & 0.18 & 150 & 0.24339 & 190 & 0.29308 \\
\hline 31 & 0.05 & 71 & & 1 & 0.18 & 151 & 0.24472 & 191 & .29424 \\
\hline 32 & 0.05 & 72 & 0 & 112 & 0.18 & 152 & 0.24604 & 192 & 0.29539 \\
\hline 30 & & 73 & & 113 & 0. & 153 & .24736 & 193 & 0.29654 \\
\hline 34 & 0.0 & 74 & & 114 & 0.19 & 154 & 67 & 194 & 0.29768 \\
\hline 35 & 0.06 & 75 & 0 & 5 & 019 & 155 & 98 & 195 & 0.29882 \\
\hline 36 & $0.064^{\prime}$ & 76 & 0 & 116 & 0.19 & 156 & 28 & 196 & 0.29996 \\
\hline 37 & 0.066 & 77 & & 117 & 10 & 157 & 0.25 & 197 & 30109 \\
\hline 38 & 0.068 & 78 & 0.136 & 8 & 0.198 & 158 & 0.25387 & 198 & 0.30222 \\
\hline 39 & 0.07006 & 79 & & 119 & 00 & 159 & 0.25517 & 199 & 0.30335 \\
\hline 40 & 0.071826 & 80 & 0.13978 & 120 & 0.20159 & 160 & 0.25645 & 200 & 0.30447 \\
\hline
\end{tabular}

Table 2: $\mu_{1}(j \pi / 1000)$ vs. $j, j=1, \ldots, 200$, by step 1 . 


\begin{tabular}{|c|c|c|c|c|c|c|c|}
\hline 205 & 0.31001 & 405 & 0.4667 & 605 & 0.54346 & 805 & 0.57978 \\
\hline 210 & 0.31546 & 410 & 0.46936 & 610 & 0.54476 & 810 & 0.58031 \\
\hline 215 & 0.32081 & 415 & 0.47196 & 615 & 0.54604 & 815 & 0.58084 \\
\hline 220 & 0.32607 & 420 & 0.47453 & 620 & 0.54729 & 820 & 0.58135 \\
\hline 225 & 0.33123 & 425 & 0.47704 & 625 & 0.54852 & 825 & 0.58185 \\
\hline 230 & 0.3363 & 430 & 0.47951 & 630 & 0.54973 & 830 & 0.58233 \\
\hline 235 & 0.34129 & 435 & 0.48194 & 635 & 0.55092 & 835 & 0.58281 \\
\hline 240 & 0.34618 & 440 & 0.48433 & 640 & 0.55208 & 840 & 0.58328 \\
\hline 245 & 0.35098 & 445 & 0.48667 & 645 & 0.55323 & 845 & 0.58372 \\
\hline 250 & 0.3557 & 450 & 0.48897 & 650 & 0.55435 & 850 & 0.58415 \\
\hline 255 & 0.36034 & 455 & 0.49124 & 655 & 0.55545 & 855 & 0.58458 \\
\hline 260 & 0.36489 & 460 & 0.49346 & 660 & 0.55653 & 860 & 0.58499 \\
\hline 265 & 0.36936 & 465 & 0.49565 & 665 & 0.55758 & 865 & 0.5854 \\
\hline 270 & 0.37375 & 470 & 0.49779 & 670 & 0.55862 & 870 & 0.5858 \\
\hline 275 & 0.37806 & 475 & 0.4999 & 675 & 0.55964 & 875 & 0.58619 \\
\hline 280 & 0.38229 & 480 & 0.50197 & 680 & 0.56064 & 880 & 0.58636 \\
\hline 285 & 0.38645 & 485 & 0.50401 & 685 & 0.56161 & 885 & 0.58669 \\
\hline 290 & 0.39053 & 490 & 0.50601 & 690 & 0.56257 & 890 & 0.58701 \\
\hline 295 & 0.39454 & 495 & 0.50798 & 695 & 0.56351 & 895 & 0.58733 \\
\hline 300 & 0.39848 & 500 & 0.50991 & 700 & 0.56443 & 900 & 0.58763 \\
\hline 305 & 0.40235 & 505 & 0.5118 & 705 & 0.56533 & 905 & 0.58793 \\
\hline 310 & 0.40614 & 510 & 0.51367 & 710 & 0.56622 & 910 & 0.58821 \\
\hline 315 & 0.40987 & 515 & 0.5155 & 715 & 0.56708 & 915 & 0.5885 \\
\hline 320 & 0.41354 & 520 & 0.5173 & 720 & 0.56793 & 920 & 0.58877 \\
\hline 325 & 0.41713 & 525 & 0.51907 & 725 & 0.56876 & 925 & 0.58904 \\
\hline 330 & 0.42067 & 530 & 0.52081 & 730 & 0.56957 & 930 & 0.58931 \\
\hline 335 & 0.42413 & 535 & 0.52251 & 735 & 0.57037 & 935 & 0.58956 \\
\hline 340 & 0.42754 & 540 & 0.52419 & 740 & 0.57115 & 940 & 0.58956 \\
\hline 345 & 0.43089 & 545 & 0.52584 & 745 & 0.57191 & 945 & 0.58978 \\
\hline 350 & 0.43418 & 550 & 0.52745 & 750 & 0.57265 & 950 & 0.59 \\
\hline 355 & 0.4374 & 555 & 0.52904 & 755 & 0.57338 & 955 & 0.59024 \\
\hline 360 & 0.44058 & 560 & 0.5306 & 760 & 0.57409 & 960 & 0.59049 \\
\hline 365 & 0.44369 & 565 & 0.53214 & 765 & 0.57479 & 965 & 0.59077 \\
\hline 370 & 0.44675 & 570 & 0.53364 & 770 & 0.57547 & 970 & 0.59113 \\
\hline 375 & 0.44976 & 575 & 0.53512 & 775 & 0.57614 & 975 & 0.59149 \\
\hline 380 & 0.45271 & 580 & 0.53658 & 780 & 0.57679 & 980 & 0.59143 \\
\hline 385 & 0.45561 & 585 & 0.538 & 785 & 0.57743 & 985 & 0.59131 \\
\hline 390 & 0.45846 & 590 & 0.5394 & 790 & 0.57805 & 990 & 0.59114 \\
\hline 395 & 0.46125 & 595 & 0.54078 & 795 & 0.57865 & 995 & 0.59092 \\
\hline 400 & 0.464 & 600 & 0.54213 & 800 & 0.57922 & 1000 & 0.59064 \\
\hline
\end{tabular}

Table 3: $\mu_{1}(j \pi / 1000)$ vs. $j, j=205, \ldots, 1000$, by step 5 . 